University of Wollongong

Research Online

Faculty of Engineering and Information

Faculty of Engineering and Information

Sciences - Papers: Part B

Sciences

2019

Grain boundary induced deformation mechanisms in nanocrystalline Al by molecular dynamics simulation: From interatomic potential perspective

\author{
Liang Zhang \\ University of Tokyo, liangz@uow.edu.au \\ Yasushi Shibuta \\ University of Tokyo \\ Xiaoxu Huang \\ Chongqing University \\ Cheng Lu \\ University of Wollongong, chenglu@uow.edu.au \\ Mao Liu \\ ml818@uowmail.edu.au
}

Follow this and additional works at: https://ro.uow.edu.au/eispapers1

Part of the Engineering Commons, and the Science and Technology Studies Commons

Research Online is the open access institutional repository for the University of Wollongong. For further information contact the UOW Library: research-pubs@uow.edu.au 


\title{
Grain boundary induced deformation mechanisms in nanocrystalline Al by molecular dynamics simulation: From interatomic potential perspective
}

\author{
Abstract \\ Nanocrystalline metals exhibit many excellent mechanical properties and their underlying deformation \\ mechanisms have been studying widespread. The well-designed atomistic simulations can predict the \\ mechanical behavior of materials ahead of experiments and provide sufficient information on the atomic \\ scale. The choice of appropriate interatomic potential is one of the main concerns of any atomistic \\ simulation that needs to be seriously considered in order to obtain reliable results. In this study, we \\ investigated the mechanical response and the deformation mechanisms of nanocrystalline Al under \\ uniaxial loading by molecular dynamics (MD) simulations with different embedded atom method (EAM) \\ interatomic potentials. The selection of potential has a significant influence on the simulation result, and \\ the reliability of these potentials was evaluated based on the available experimental data in the literature. \\ In the elastic stage, the stress-strain response and Young's modulus of the simulated samples varied with \\ different potentials. Three independent elastic constants of single crystal Al were used to predict the \\ isotropic elastic modulus of the polycrystal Al sample. In the plastic stage, multiple grain boundary (GB) \\ induced deformation mechanisms were observed, including GB migration, intergranular fracture, \\ dislocation nucleation from GB, and deformation twinning. The generalized stacking fault energies of $\mathrm{Al}$ \\ were calculated by MD simulation using different potentials, and their effects on the dislocation \\ nucleation and deformation twinning mechanisms were discussed. This work signifies the important role \\ of GBs play in nanocrystalline metals during plastic deformation and highlights the significance of using \\ the appropriate interatomic potential for a specific simulation problem.

\section{Disciplines} \\ Engineering | Science and Technology Studies

\section{Publication Details} \\ Zhang, L., Shibuta, Y., Huang, X., Lu, C. \& Liu, M. (2019). Grain boundary induced deformation mechanisms \\ in nanocrystalline Al by molecular dynamics simulation: From interatomic potential perspective. \\ Computational Materials Science, 156 421-433.
}

This journal article is available at Research Online: https://ro.uow.edu.au/eispapers1/2044 


\title{
Grain boundary induced deformation mechanisms in nanocrystalline Al by molecular dynamics simulation: from interatomic potential perspective
}

\author{
Liang Zhang ${ }^{a}$, Yasushi Shibuta ${ }^{a}$, Xiaoxu Huang ${ }^{b}$, Cheng Lu ${ }^{c}$, Mao Liu ${ }^{d}$ \\ ${ }^{a}$ Department of Materials Engineering, The University of Tokyo, Bunkyo-ku, \\ Tokyo 113-8656, Japan \\ ${ }^{b}$ College of Materials Science and Engineering, Chongqing University, \\ Chongqing, 400044, China \\ ${ }^{c}$ Faculty of Engineering and Information Science, University of Wollongong, \\ Wollongong, NSW 2522, Australia \\ ${ }^{d}$ Faculty of Science, Engineering and Technology, Swinburne University of Technology, \\ Melbourne, VIC 3122, Australia
}

\begin{abstract}
Nanocrystalline metals exhibit many excellent mechanical properties and their underlying deformation mechanisms have been studying widespread. The well-designed atomistic simulations can predict the mechanical behavior of materials ahead of experiments and provide sufficient information on the atomic scale. The choice of appropriate interatomic potential is one of the main concerns of any atomistic simulation that needs to be seriously considered in order to obtain reliable results. In this study, we investigated the mechanical response and the deformation mechanisms of nanocrystalline Al under uniaxial loading by molecular dynamics (MD) simulations with different embedded atom method (EAM) interatomic potentials. The selection of potential has a significant influence on the simulation result, and the reliability of these potentials was evaluated based on the available experimental data in the literature. In the elastic stage, the stress-strain response and Young's modulus of the simulated samples varied with different potentials. Three independent elastic constants of single crystal Al were used to predict the isotropic elastic modulus of the polycrystal Al sample. In the plastic stage, multiple grain boundary (GB) induced deformation mechanisms were observed, including GB migration, intergranular fracture, dislocation nucleation from GB, and deformation twinning. The generalized stacking fault energies of $\mathrm{Al}$ were calculated by MD simulation using different potentials, and their effects on the dislocation nucleation and deformation twinning mechanisms were discussed. This work signifies the important role of GBs play in nanocrystalline metals during plastic deformation and highlights the significance of using the appropriate interatomic potential for a specific simulation problem.
\end{abstract}

Keywords: Molecular dynamics; Interatomic potential; Grain boundary; Plastic deformation

Corresponding author. liang@mse.mm.t.u-tokyo.ac.jp (L. Zhang)

\section{Introduction}


Nanomaterials have demonstrated many unique mechanical, chemical and physical properties which have attracted wide attention in the past few decades. Nanocrystalline metals usually exhibit ultra-high strength, superior hardness and enhanced wear resistance due to the nanoscale and interface effect [1-5]. Because of their excellent mechanical properties and the great potential for engineering applications, a large effort has been devoted to investigating the relationship between their structures, mechanical response and deformation mechanisms [6-9]. However, the atomic scale is an area hardly accessible experimentally since the extreme small grain size leads to additional difficulties to observe the deformation details on the nanometer scale by means of experiment. Facilitated by rapid increases in computational power, atomistic simulations are served as a useful tool for analyzing and predicting various fundamental static and dynamic properties of materials. For example, the first-principles calculation is considered as the most accurate theoretical method of investigating the properties of materials at the atomic level. However, it is insufficient to study the dynamic behaviors because the size (or the number of atoms) it can deal with is very limited. Molecular dynamics (MD) simulation based on semi-empirical interatomic potential can usually simulate millions or up to billion of atoms [10]. Therefore, it was used extensively to study the mechanical properties of materials, and great progress has been made in simulating and understanding deformation mechanisms $[5,11,12]$. Also, recent post-processing technologies such as the visualization tools $[13,14]$ and the sophisticated structure identification methods $[15,16]$ have improved considerably in recent years, providing more information on the atomic scale.

MD simulation is described by interatomic potential (or force field) that used to model the interactions of the atoms in the simulation. Therefore, the selection of interatomic potential has a crucial impact on the simulation result, that is, the reliability of simulations dependent entirely on the reality and accuracy of the selected potential. Many interatomic potentials have been developed for metallic materials and their alloys, and the most widely used in MD simulations is the embedded atom method (EAM) $[17,18]$. Also, the modified embedded atom method (MEAM) was developed by considering the directionality of bonding [19]. More information can be referenced to the websites of NIST Interatomic Potentials Repository (NIST-IPR) [20, 21] and the Knowledgebase of Interatomic Models (OpenKIM) [22], which provide different types of interatomic potential for various materials. To accurately simulate the deformation behavior of the metallic materials, the selected potential should be able to correctly reproduce various fundamental physical properties of relevant elements in pure metals or multicomponent alloys. For example, the elastic constants reflect the mechanical response of materials in the elastic deformation stage, which have a significant influence on their yield strength [23]. The stacking fault energy plays a critical role in the deformation properties of materials in the stage of plastic deformation [24-26]. The stacking fault energy can affect phenomena such as the formation of perfect or partial dislocations, the ability of a dislocation to cross slip, and the formation of deformation twins, all of which have an important impact on the strength and toughness of nanostructured metals. It was realized that there is often more than one potential describing any given material. For examples, we can find dozens of interatomic potentials which are designed for the simulation of $\mathrm{Al}$ and its alloys [21, 22]. Since different interatomic potentials may give discrepant results for the same problem [20], it is important for users to choose the appropriate one to perform their desired simulations.

In this study, we take $\mathrm{Al}$ as an example to investigate its mechanical response and deformation mechanisms of nanocrystalline metals under uniaxial tension by using MD simulations. There are 
two main purposes of this work. Firstly, we studied GB-induced deformation mechanisms in nanocrystalline Al. When the grain sizes are reduced to the nanometer scale, the traditional deformation mechanisms based on lattice dislocation are replaced gradually by GB mediated processes [24]. These GB-induced mechanisms are extensively reported and discussed in this study. Secondly, we examined different interatomic potentials of $\mathrm{Al}$ and evaluated these potentials by comparing the simulation results and the available experimental result. The article is organized as follows. Section 2 introduces model construction and the simulation methods. Section 3 reports the mechanical responses of nanocrystalline Al with different interatomic potentials. In section 4, different GB-induced mechanisms in the stage of plastic deformation are presented. We discuss the simulation results in section 5 and conclude the work in section 6 .

\section{Simulation method}

The nanocrystalline Al sample with a columnar grain structure was created using the Voronoi construction procedure. The simulation sample contains six grains and they are rotated around the $[001] / z$ axis with particular misorientation angles. Therefore, all the GBs in the sample are flat and have a tilt character. The distribution of the grain seeds was artificially controlled in order to obtain a uniform grain size. The dimensions of the simulation box were set to $486 \AA, 486 \AA$, and $81 \AA$ along the $\mathrm{x}, \mathrm{y}$, and $\mathrm{z}$ directions, respectively. Periodic boundary conditions were applied in all directions. The total number of atoms is about 1,200,000, and the average grain size is $22.4 \mathrm{~nm}$. The simulation sample generated by the Voronoi method usually leads to the overlap of adjacent grains, which contain atoms that are physically too close in the boundary area. Followed the previous method that was used to construct the bicrystal model [27], we defined a critical distance to delete the atoms with an unphysically short distance from others and ensure that the first nearest neighbor atoms are not found within the cutoff distance. The initial configuration of the simulation sample was shown in Fig.1(a), where atoms are colored according to their common neighbor analysis (CNA) value. The blue atoms represent the fcc atoms inside the grain, and the red atoms represent the GBs between the grains. To highlight the GB structure, fcc atoms were removed and viewed along the axis of rotation. The misorientation angles of the $16 \mathrm{GBs}$ in the nanocrystalline sample were indicated, as shown in Fig.1(b).

To obtain the equilibrium structure of the GBs, the initial sample was equilibrated by an energy minimization procedure with a standard conjugate-gradient algorithm at $0 \mathrm{~K}$, as shown in Fig.1(c) and (d). For clear expression, GBs were indicated as $\mathrm{GB}_{\mathrm{nm}}$ in the following work, where $\mathrm{n}$ and $\mathrm{m}$ are the indexed numbers of any two neighbored grains. Due to the symmetry of fcc lattice, GB with misorientation angle close to $0^{\circ}$ or $90^{\circ}$ was referred to the low-angle $\mathrm{GB}$ around the $<100>$ tilt axis; this is the case for $\mathrm{GB}_{23}\left(\theta=4^{\circ}\right), \mathrm{GB}_{56}\left(\theta=14.3^{\circ}\right), \operatorname{GB}_{35}\left(\theta=16.6^{\circ}\right)$, and $\mathrm{GB}_{14}\left(\theta=73.4^{\circ}\right)$. The discrete and continuous lines of GB atoms in the energy-minimized sample represent low-angle and highangle GBs respectively. The system was annealed at $300 \mathrm{~K}$ for $100 \mathrm{ps}$ in isobaric-isothermal (NPT) ensemble to achieve a zero pressure in all directions before loading. During dynamic tension, a uniaxial tensile strain was applied by continuously scaling the atomic coordinates and box size along $\mathrm{x}$ - or $\mathrm{y}$-direction with a constant strain rate of $5 \times 10^{8} \mathrm{~s}^{-1}$, while the pressure in the other two directions were kept at zero. The simulation temperature was maintained at $300 \mathrm{~K}$ and an integration time step was set as $1 \mathrm{fs}$ throughout the simulations. Simulations were performed using the parallel molecular dynamics package LAMMPS [28], and the visualization tools Ovito [14] were used to illustrate the 
sample.

To highlight the influence of the interatomic potential on the simulation results, different EAM and MEAM basis interatomic potentials for $\mathrm{Al}$ and $\mathrm{Al}$ alloy were examined [29-38]. EAM potentials are widely used in the MD simulations for metals and their alloys. There are many different EAM based interatomic potentials developed or optimized for a given element by considering its mechanical, chemical or physical properties. In addition, we noticed that while potentials developed for a single element can reflect some properties of the element very well, it may not be suitable for simulating other characteristics. For example, the potential of Al developed by Ercolessi and Adams [39] can show an excellent thermal and surface properties of Al, but it cannot accurately describe the stacking fault energy of $\mathrm{Al}$; this potential was modified in their later work by using a slightly larger cut-off [40]. On the other hand, some interatomic potentials designed for the alloy system can well express the properties of the individual component. For example, the potentials developed by Mishin et al. for Ni-Al [33], Ti-Al [34] alloy systems (indexed as P5 and P6 in the follows) were constructed by the cross-interaction of the pure Al, Ni and Ti potentials. Therefore, they can reproduce the optimized fitting parameters and the relevant properties of each single component. To verify this, we have compared the Ni-Al, Ti-Al alloy potentials with the single Al potential [41] which is also developed by Mishin et al., both of which give the very similar simulation results. Therefore, some potentials which are designed for the description of Al alloy are also examined in this study. All the tested potentials were downloaded from the database of NIST-IPR [21], and some of their computed properties are listed in Table-1.

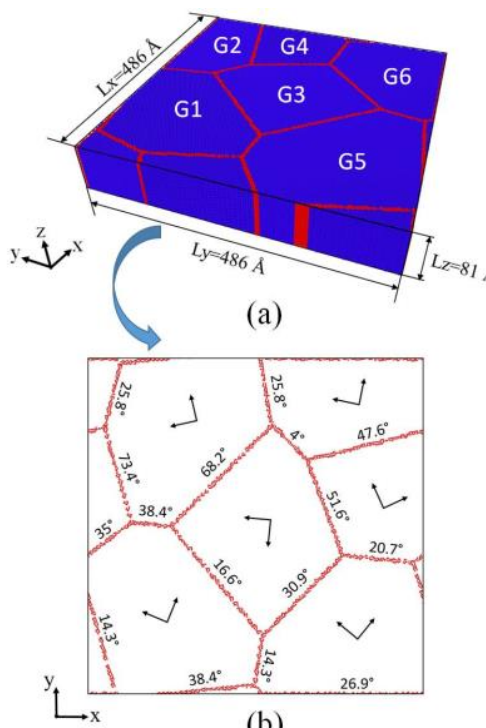

(b)

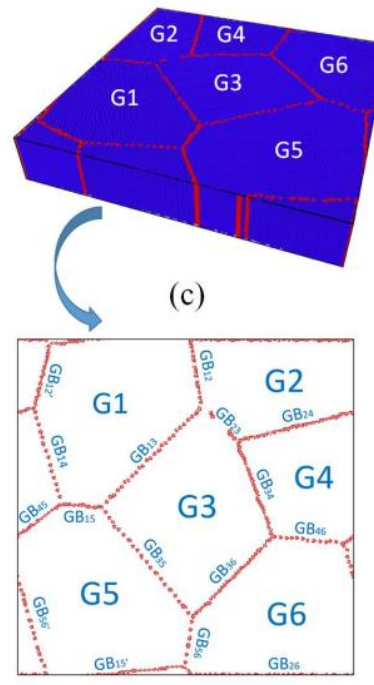

(d)

Fig.1 The nanocrystalline Al sample with six grains (G1 G6), the average grain size is $22.4 \mathrm{~nm}$. (a) The initial configuration of simulation sample, (b) fcc atoms are removed in (a) and viewed along z-direction. (c) The equilibrium configuration of simulation sample after system minimization, (d) fcc atoms are removed in (c) and viewed along z-direction. Atoms are colored according to the common neighbor analysis (CNA), where the blue atoms represent the fcc atoms inside the grain, and the red atoms represent the GBs between the grains.

Table 1. The interatomic potentials (P1 P10) of $\mathrm{Al}$ used in this study and their computed properties, including cohesive energy $\left(\mathrm{E}_{\mathrm{coh}}\right)$, lattice constant $\left(\mathrm{a}_{0}\right)$, and elastic constants $\left(\mathrm{C}_{11}, \mathrm{C}_{12}\right.$, and $\left.\mathrm{C}_{44}\right)$. The data are referenced from potential database NIST-IPR [21] and OpenKIM [22]. The experimental data (Exp.) are also included for comparison. 


\begin{tabular}{|c|c|c|c|c|c|c|}
\hline $\begin{array}{c}\text { Potential } \\
\text { ID }\end{array}$ & Potential name & $E_{\text {coh }}(e V)$ & $\mathbf{a}_{0}(\AA)$ & $\mathrm{C}_{11}(\mathrm{GPa})$ & $\mathrm{C}_{12}(\mathrm{GPa})$ & $\mathrm{C}_{44}(\mathrm{GPa})$ \\
\hline $\mathrm{P} 1{ }^{[29]}$ & AlU.meam & 3.3600 & 4.0500 & 113.5 & 61.6 & 45.4 \\
\hline $\mathrm{P} 2^{[30]}$ & Jelinek_2012_meam.alsimgcufe & 3.3530 & 4.0500 & 110.53 & 60.90 & 28.39 \\
\hline P3 [31] & All.eam.fs & 3.4107 & 4.0453 & 105.09 & 59.46 & 30.66 \\
\hline $\mathrm{P} 4{ }^{[32]}$ & Al-Mg.eam.fs & 3.4107 & 4.0453 & 110.18 & 61.37 & 32.56 \\
\hline $\mathrm{P} 5{ }^{[33]}$ & NiAl.eam.alloy & 3.3600 & 4.0500 & 116.81 & 60.11 & 31.66 \\
\hline $\mathrm{P} 6^{[34]}$ & Zope-Ti-Al-2003.eam.alloy & 3.3600 & 4.0500 & 116.81 & 60.11 & 31.66 \\
\hline P7 [35] & alpb-setfl.eam.alloy & 3.3593 & 4.0310 & 107.91 & 56.91 & 32.86 \\
\hline P8 ${ }^{[36]}$ & Al.set & 3.5800 & 4.0502 & 112.28 & 63.80 & 30.93 \\
\hline P9 [37] & Al_wkg_MSMSE_2009.set & 2.6460 & 4.0248 & 113.76 & 61.71 & 31.25 \\
\hline $\mathrm{P} 10^{[38]}$ & AlCu.eam.alloy & 3.3183 & 4.0498 & 90.15 & 70.73 & 33.16 \\
\hline Exp. $^{[42]}$ & & & & 107.3 & 60.08 & 28.3 \\
\hline
\end{tabular}

\section{Mechanical response}

Fig. 2 shows the stress-strain response of the nanocrystalline $\mathrm{Al}$ sample under uniaxial tension at 300 $\mathrm{K}$ with different interatomic potentials (P1 P10). It is found that some of the potentials have similar mechanical responses, while the stress responses of other potentials are quite different in both elastic and plastic deformation stages. In general, the stress first increases with the applied tensile strain until it reaches the maximum stress, after which the stress drops and then fluctuates around a mean value. Visualization of the MD result indicates that the onset of plastic deformation corresponds to dislocation nucleation and GB related activities, and it occurs at a strain very close to the maximum stress. Therefore, we defined the maximum tensile stress as the yield stress in this study. The stress curve reaches a stable stage after $15 \%$ strain, so the average stress in the strain interval from $15 \%$ to $40 \%$ deformation is defined as the flow stress.

The Young's modulus $(E)$ for each studied case is obtained from the linear portion of its corresponding stress-strain between 0 and $0.5 \%$ strain. The calculated values of Young's modulus by different potentials are plotted in Fig.3(a). We compared the result from MD simulation with the experimental data. The elastic properties of cubic single crystal can be characterized in terms of three independent elastic stiffness constants $\left(\mathrm{C}_{11}, \mathrm{C}_{12}\right.$, and $\left.\mathrm{C}_{44}\right)$. The values of the three constants for single crystal of $\mathrm{Al}$ by experiment measurement at $300 \mathrm{~K}$ are $107.3 \mathrm{GPa}, 60.08 \mathrm{GPa}$, and 28.3 GPa respectively [42]. The anisotropic single-crystal elastic constants can be converted into isotropic polycrystalline elastic modulus by using the Voigt-Reuss-Hill approximation [43]. This approach combines the upper and lower bounds by assuming the average of values obtained through the Voigt and Reuss averaging methods [44]. Specifically, the upper bound (Voigt) of shear modulus is given by

$$
G_{V}=\frac{C_{11}-C_{12}+3 C_{44}}{5}
$$

and the lower bound (Reuss) is given by

$$
G_{R}=\frac{5 C_{44}\left(C_{11}-C_{12}\right)}{4 C_{44}+3\left(C_{11}-C_{12}\right)}
$$


According to the Hill empirical average, the isotropic shear modulus can be obtained by

$$
G=\frac{G_{V}+G_{R}}{2}
$$

The Bulk modulus is defined as

$$
B=\frac{C_{11}+2 C_{12}}{3}
$$

Therefore, the Young's modulus $(E)$ can be calculated by the shear modulus $(G)$ and Bulk modulus $(B)$ by the well-defined isotropic relation

$$
E=\frac{9 G B}{3 B+G}
$$

From the above equations, the estimated Young's modulus for polycrystal $\mathrm{Al}$ is $70.8 \mathrm{GPa}$. We note that the values obtained from simulations are generally smaller than the predicted value from the experiment data, as shown in Fig.3(a). This is mainly due to the microstructure of nanocrystalline metals, in which the volume fraction of GBs is significantly increased by nanoscale grains. The less ordered structure of GBs led to the decrease of the elastic modulus of the bulk material. The model size and the limited grain numbers of the simulation sample may also result in the deviation from the predicted value based on the macroscopic experiment. As a further note, while most of the potentials gave similar values of Young's modulus, ranging from $57.8 \mathrm{GPa}$ to $66.04 \mathrm{GPa}$, there are two clear outliers (P8 and P10). Obviously, the two potentials can not accurately show the mechanical properties of nanocrystalline $\mathrm{Al}$.
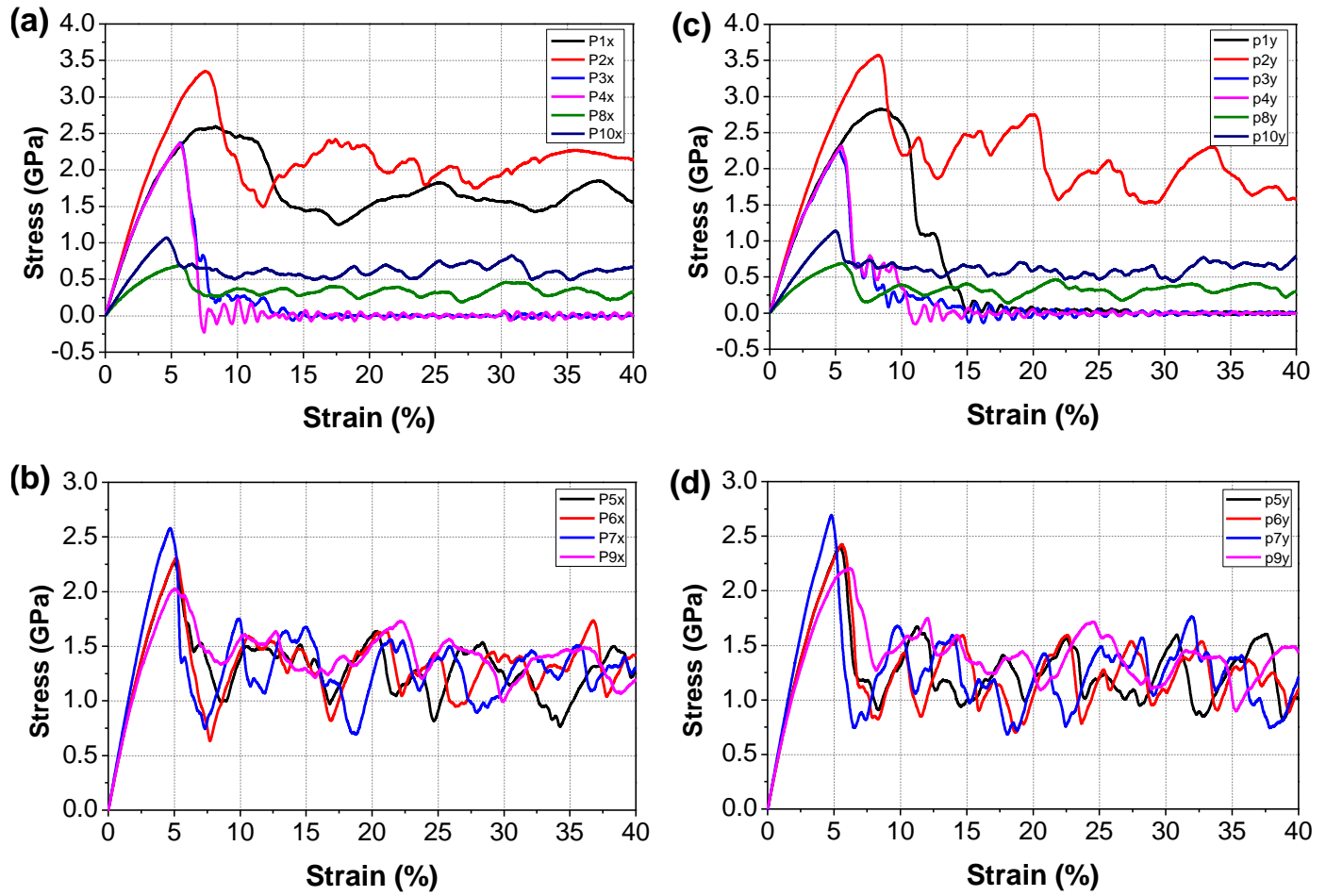

Fig.2 Stress-strain curve of nanocrystal Al samples under uniaxial tension along x-direction (a-b) and y-direction (cd). For each loading direction, the stress-strain curves by different interatomic potentials are divided into two groups for clear presentation. 
The yield stress, yield strain, and flow stress of the nanocrystal Al sample with different potentials are plotted in Fig.3(b) to 3(d), respectively. As expected, the lower Young's modulus obtained by P8 and P10 resulted in the lower yield stress of the simulation sample. The flow stress shows a similar trend as the yield stress except for the P3 and P4 cases, and the P1 case in y-direction. The flow stress value of zero indicates a brittle fracture of the sample during the dynamic tension. The yield strain represents the onset of the plastic deformation, which was achieved when dislocation and some GB associated mechanisms were activated. The detail information will be presented in the next section. From the simulation results, it is clear to see that different potentials, for what is nominally the same material, can show distinct mechanical responses.

(a)
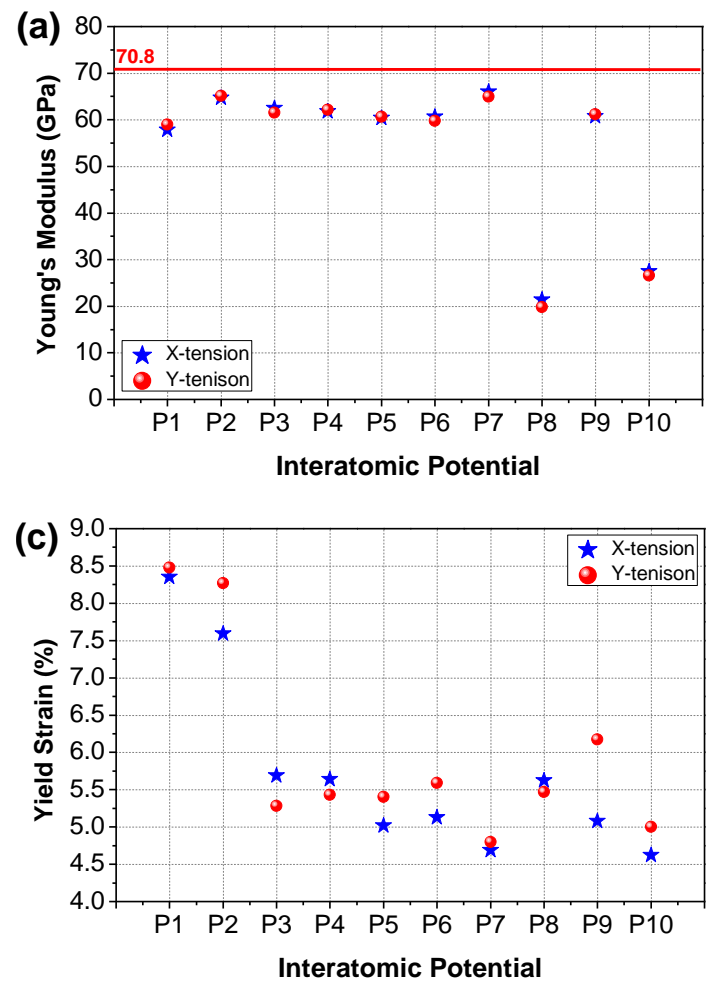

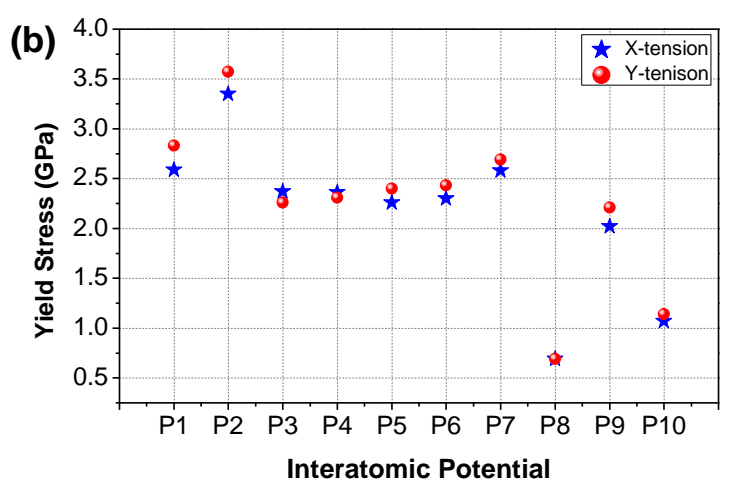

(d)

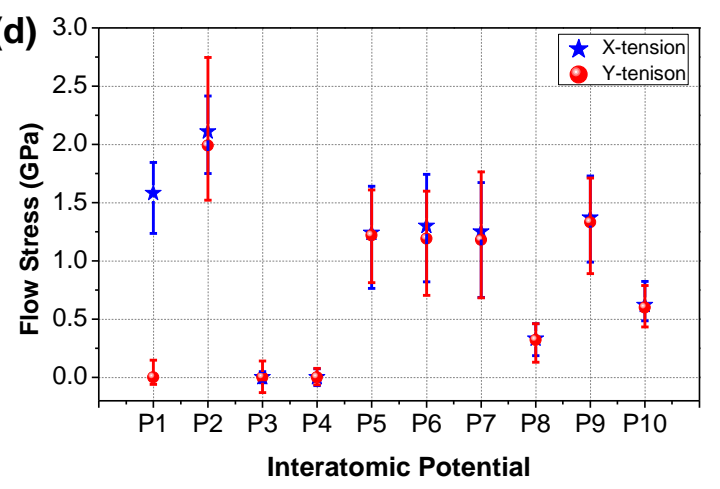

Fig.3 Stress-strain response of nanocrystal Al samples with different interatomic potentials (P1 P10). (a) Young's modulus, (b) yield stress, (c) yield strain, and (d) flow stress. The error bar in (d) indicates the maximum and minimum stress of the stress flow between $15 \%$ to $40 \%$ strain.

\section{Deformation mechanisms}

Dislocation generation via Frank-Read sources in conventional coarse-grained metals is significantly hampered or even completely suppressed when the grain size decreases to the order of $10 \mathrm{~nm}$ in metallic nanomaterials [3]. We note that the $\sim 20 \mathrm{~nm}$ grain size in this study is still too small to support dislocation multiplication through Frank-Read source type mechanisms. Therefore, the conventional deformation mechanisms based on nucleation and movement of lattice dislocation are replaced by GB mediated processes. Fig. 4 and Fig. 5 show the MD simulation snapshots of the nanocrystalline $\mathrm{Al}$ samples after $40 \%$ tensile deformation along $\mathrm{x}$ - and $\mathrm{y}$-direction using different interatomic potentials. It was found that the simulation results varied with the applied potentials, from the brittle fracture at GBs to GB-induced deformation mechanisms in plastic deformation stage, 
including the 'pure' GB mechanisms by the motion of GBs themselves and the dislocation activities that associated to GBs. Some typical GB-mediated mechanisms are presented in the follows.
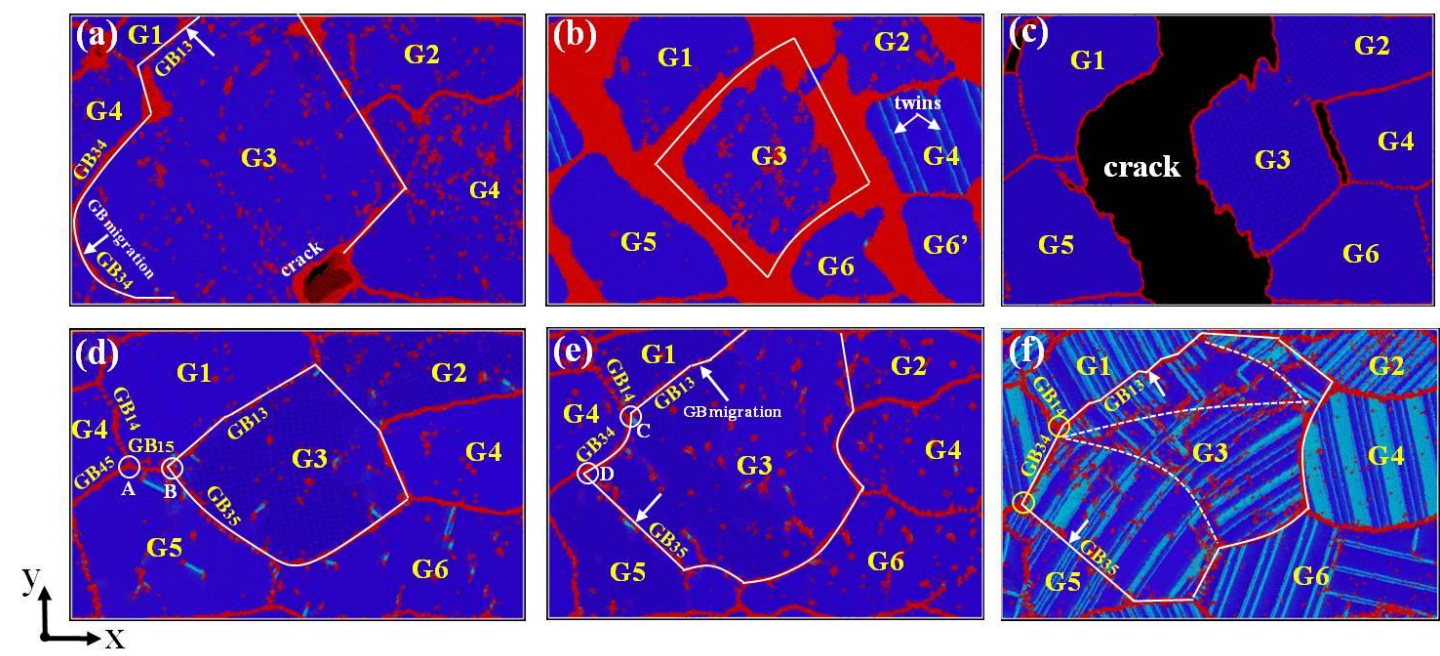

Fig.4 Snapshots of nanocrystal Al sample after $40 \%$ tensile deformation along $\mathrm{x}$-direction by different interatomic potentials. (a)-(f) corresponds to the result from the potentials of P1, P2, P3, P5, P9 and P10. Atoms are colored according to their CNA values. The atoms with fcc structure are colored in dark blue; GBs and dislocation cores are colored in red. The atoms with hcp structure are colored in light blue, a single layer of hcp atoms represents twin boundary, and the continuous hcp atom layers represents the stacking fault. The white solid lines highlight the grain size of G3, and the white dotted lines in (f) indicate the sub-GBs in G3 that generated by the movement and intersection of dislocations.

\subsection{GB migration}

Experimental observations and computer simulations have shown that stress-induced GB migration is an important phenomenon that initiates plastic deformation of grains [45-49]. For example, a significant grain growth mediated by GBs migration during tensile tests at the high stress was observed in nanocrystalline $\mathrm{Al}$ film at room temperature [50]. In our simulations, GB migration has been observed in several cases with different interatomic potentials. We first compare the results in the cases of P5, P9, and P10. The GB network did not change essentially after $40 \%$ deformation in P5 case, each grain was just elongated along the tensile direction, as shown in Fig.4(d) and Fig.5(d). However, in the cases of $\mathrm{P} 9$ and $\mathrm{P} 10, \mathrm{~GB}_{13}$ and $\mathrm{GB}_{35}$ moved towards the outside of $\mathrm{G} 3$ under $\mathrm{x}$ tension and moved in an opposite direction under y-tension, resulting in the expansion or shrink of G3 and the surrounding grains. For clarity, the grain size of G3 is circled by the white solid lines in the figures.

The local GB network has been changed as the migration of GBs. For instance, there are two triple junctions which indexed ' $A$ ' and 'B' in Fig.4(d), which were initially surrounded by grains of G1-G4-G5 and G1-G3-G5 and connected by $\mathrm{GB}_{15}$. After the migration of $\mathrm{GB}_{13}$ and $\mathrm{GB}_{35}, \mathrm{~GB}_{15}$ has disappeared and the triple junctions $\mathrm{A}$ and $\mathrm{B}$ have now changed to $\mathrm{C}$ and $\mathrm{D}$ which surrounded by G1-G3-G4 and G3-G4-G5 and connected by GB 34 , as shown in Fig.4(e). On the other hand, more obvious migration of $\mathrm{GB}_{13}$ and $\mathrm{GB}_{34}$ under tension was observed in the case of $\mathrm{P} 1$. The fast movement of $\mathrm{GB}_{34}$ led to the significant growth of $\mathrm{G} 3$ and the complete disappearance of G5, as shown in Fig.4(a). Note that the initial flat boundary plane of $\mathrm{GB}_{34}$ became severe curved after its 
migration. This is different from previous MD simulations of bicrystal Al samples, where the [001] tilt GBs can maintain a flat boundary plane during the migration process [51-54]. The difference is that in the current polycrystal sample, each grain is embedded in the surrounding grains and the adjacent GBs are connected to form a triple junction structure. While the middle segment of $\mathrm{GB}_{34}$ can move freely under stress, the migration at both ends of $\mathrm{GB}_{34}$ was impeded by the triple junctions, leading to the bending of the entire boundary. In addition, it was noted that a crack was nucleated at $\mathrm{GB}_{56}$ during tension along $\mathrm{x}$-direction. Since a substantial grain growth is usually observed in regions with high local stresses, the fast migration of $\mathrm{GB}_{35}$ may be facilitated by the adjacent crack tip which serves as an effective stress concentration.
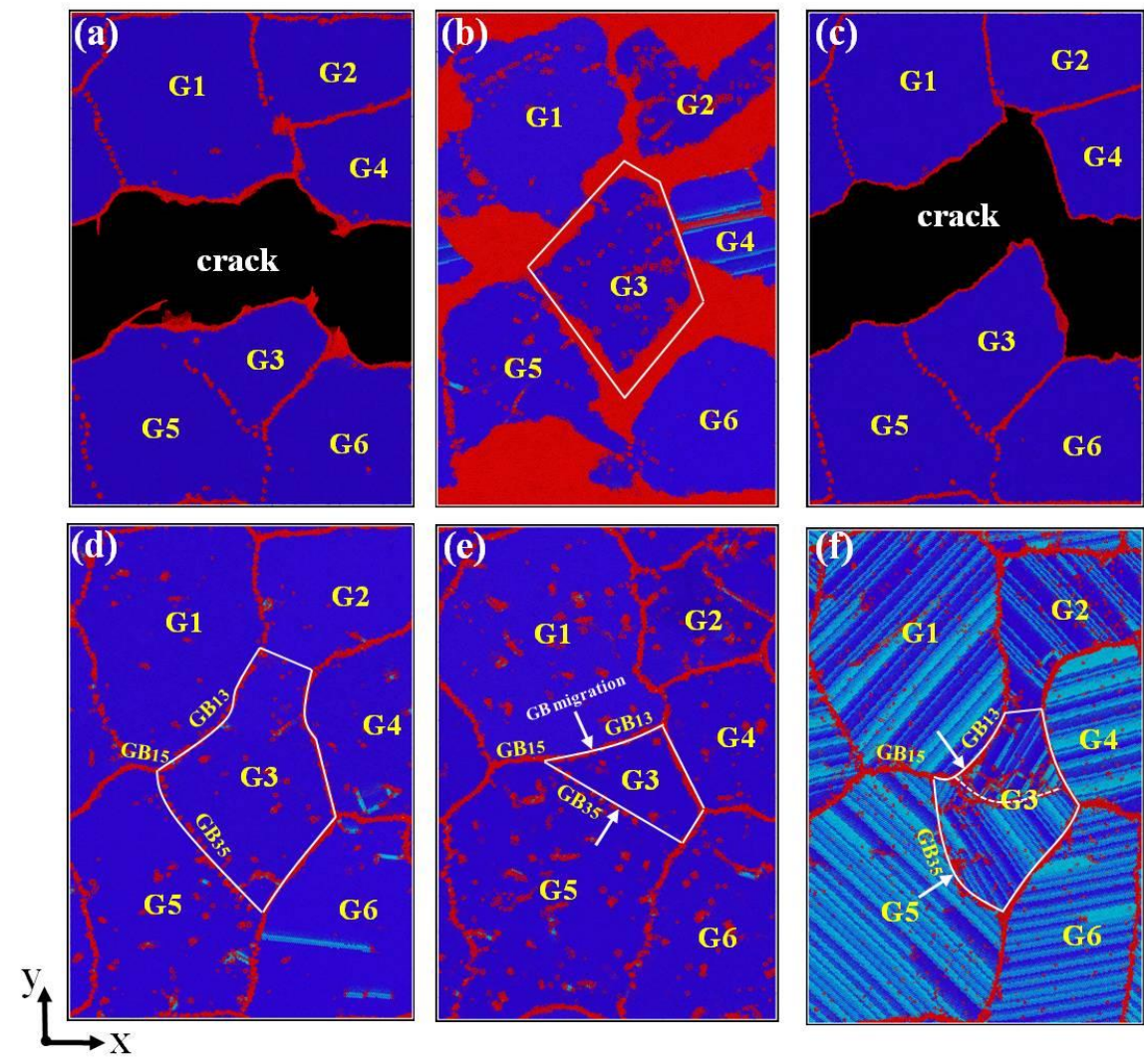

Fig.5 Snapshots of nanocrystal Al sample after $40 \%$ tensile deformation along y-direction by different interatomic potentials. (a)-(f) corresponds to the result from the potentials of P1, P2, P3, P5, P9 and P10. Atoms are colored in the same manner as Fig.4.

\subsection{Intergranular fracture}

Compared with the bulk phase in grain interiors, the GBs have low atomic density and weak interatomic bonds. Therefore, cracks tend to nucleate and grow at GBs under external loading if other deformation mechanisms were not involved or these mechanisms cannot play an effective role in accommodating system stress. In this study, cracks were observed during deformation under $\mathrm{x}$ and y-tension in the P1, P3, and P4 cases. Fig.6(a) shows the nucleation and evolution of cracks in the simulation sample with $\mathrm{P} 3$ potential under x-tension. In Fig.6(a1), the first crack ' $a$ ' was observed at $\mathrm{GB}_{56}$ at about $6 \%$ strain, and two embryo cracks $b$ and $c$ were observed at $\mathrm{GB}_{35}$ and $\mathrm{GB}_{13}$, respectively. As the increase of tensile strain, the propagation of crack $a$ led to the cracking 
of $\mathrm{GB}_{12}$. Meanwhile, cracks $b$ and $c$ were dilated, and the other two cracks $d$ and $e$ appeared at $\mathrm{GB}_{35}$, as shown in Fig.6(a2). The crack tip of $c$ propagated rapidly along $\mathrm{GB}_{13}$, and other cracks also expanded with additional tensile strain. Eventually, all the independent cracks connected together which led to the formation of the main brittle crack, as shown in Fig.6(a3) and (a4). The formation of cracks in the P1 case under y-tension is shown in Fig.6(b). In a similar way, the cracks first appeared on the boundaries which are perpendicular to the loading direction, i.e. crack $a$ at $\mathrm{GB}_{15}$ and crack $b$ at $\mathrm{GB}_{46}$. Then, cracks $c, d$ and $e$ were nucleated at $\mathrm{GB}_{13}$ and $\mathrm{GB}_{34}$ in succession. The propagation and coalescence of numerous intergranular cracks led to the overall fracture of the sample. In Fig.6, dislocations were rarely observed during the deformation, and the samples exhibited a brittle fracture mode. The intergranular fracture corresponds to the sharp decrease of tensile stress to a zero stress level in Fig.3.

It is worth noting that crack was nucleated at $\mathrm{GB}_{56}$ in the P1 case under $\mathrm{x}$-tension. However, the growth of crack was suppressed, and the size of crack remains limited after $40 \%$ tensile strain (see Fig.4a). The different tensile responses and the crack behaviors between P1 and P3 under x-tension can be ascribed to the migration of $\mathrm{GB}_{35}$. Specifically, the crack nucleation at $\mathrm{GB}_{56}$ and the migration of $\mathrm{GB}_{34}$ have mutual interactions. The high-stress field around crack tips promoted the movement of $\mathrm{GB}_{34}$ under loading. On the other hand, $\mathrm{GB}$ migration played a significant role in accommodating the system stress and releasing the stress concentration at $\mathrm{GB}$, which is not conducive to the crack propagation at $\mathrm{GB}$. Also, the migration of $\mathrm{GB}_{34}$ in the vicinity of the crack at $\mathrm{GB}_{56}$ blocked the way of crack propagation along the nearby GBs. Therefore, crack growth was hindered by substantial crack blunting, which thereby gives the material higher toughness in the $\mathrm{P} 1$ case under $\mathrm{x}$-tension.

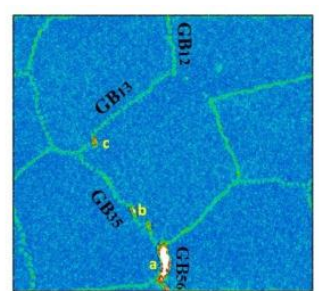

(a1) $\varepsilon=6 \%$

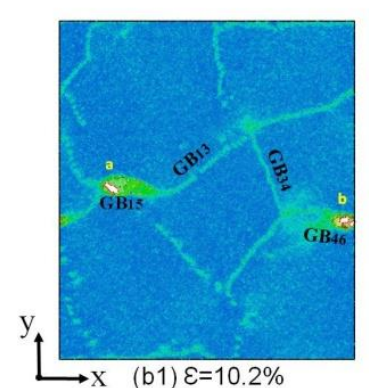

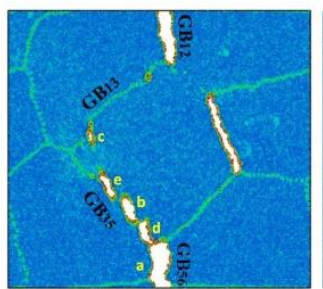

(a2) $\varepsilon=7 \%$

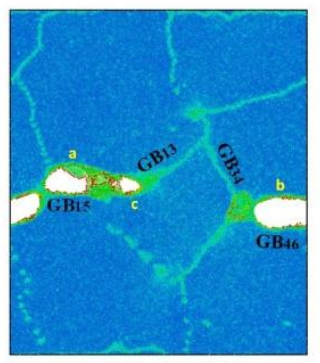

(b2) $\varepsilon=11.4 \%$

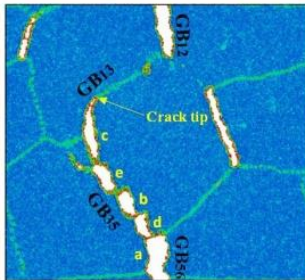

(a3) $\varepsilon=7.8 \%$

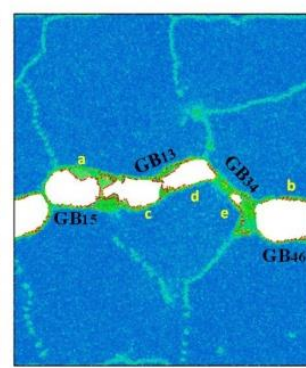

(b3) $\varepsilon=14.4 \%$

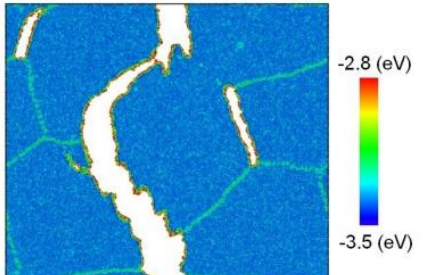

(a4) $\varepsilon=15 \%$

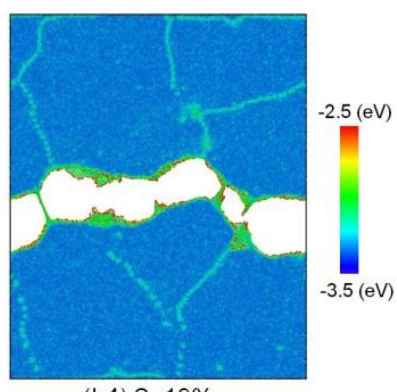

(b4) $\varepsilon=19 \%$

Fig.6 Snapshots of nanocrystal Al sample at different deformation stages in the case of $\mathrm{P} 3$ potential under $\mathrm{x}$-tension (a), and in the case of $\mathrm{P} 1$ potential under $\mathrm{y}$-tension (b). Atoms are colored according to their potential energies.

\subsection{Dislocation nucleation from $G B$}

Partial dislocation emission from grain boundaries plays a major role during deformation when the grain size decreases [55-60]. This mechanism was well represented in the simulations of nanocrystalline Al sample using different interatomic potentials. The sample constructed in this 
study is a dislocation-free system, and all the dislocations observed during deformation are exclusively emitted from the GBs or the triple junctions. Fig.7 shows the scenarios of dislocation emission at the early plastic stage under x-tension in the cases of P5, P7, P9, and P10. The structural defects and their evolution during the deformation was identified by the CNA value. In Fig.7(a1), the initial dislocation nucleation was observed at about 5\% deformation in the P5 case. A number of leading Shockley partial dislocations (L1, L2, and L3) were emitted near the G3-G4-G6 and G3G5-G6 triple junctions, leaving an intrinsic stacking fault behind where the dislocations swept. With the increase of tensile strain, extended dislocations were formed in G5 and G6, i.e. the trailing Shockley partial dislocations ( $\mathrm{T} 1$ and $\mathrm{T} 2$ ) were nucleated on the same slip plane and reduced the stacking fault that created previously by the leading partials, as shown in Fig.7(a2). The extended dislocations slipped inside of the grains as the strain build up, and the distance of stacking fault between the leading and trailing partials was reduced. When the dislocations crossed the entire grain, they interacted with the GBs on the other side and were eventually absorbed by the GBs, leaving no dislocations to accumulate inside the grain, as shown in G5 and G6 in Fig.7(a3) and (a4).

The results obtained by P7 and P9 potentials are similar to the case of P5. In the early stage of plastic deformation, dislocations were nucleated from one GB and absorbed by another. However, in the case of $\mathrm{P9}$, the leading partials and trailing partials were emitted from GBs almost simultaneously when the critical stress has been reached. Therefore, staking faults were hardly observed during the deformation process, as shown in Fig.7(b). In contrast, the slip activation took place at a smaller strain level in the case of P7, and the splitting distance between the leading and trailing partials is larger than the former two cases, as shown in Fig.7(c). Nevertheless, the nucleation and propagation of the extended dislocations are the dominant deformation mechanisms to accommodate the system stress in the cases of P5, P7and P9. The slip and interactions of dislocations inside the grain contributed to the stress flow at the plastic stage. On the other hand, a distinct different scenario was observed in the case of P10, where only the leading partial dislocations were observed during tension, the nucleation of trailing partial was not observed even at a high tensile strain. Therefore, the leading partials produced stacking faults across the grains, as shown in Fig.7(d). The distance between the leading and trailing partials depends mainly on stacking fault energy that characterized by the interatomic potentials, which will be discussed in the next section. 


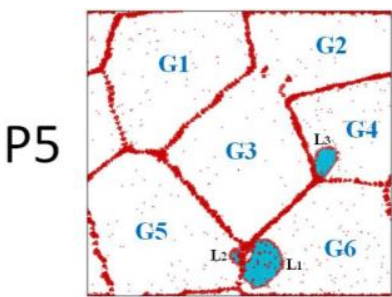

(a1) $\varepsilon=5 \%$

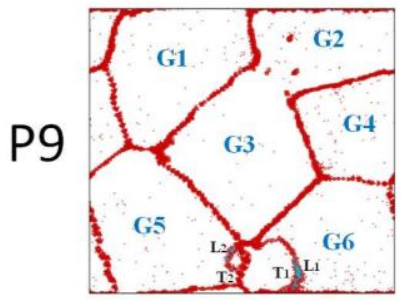

(b1) $\varepsilon=5 \%$

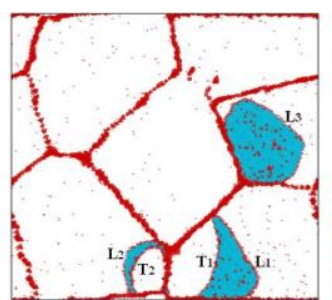

(a2) $\varepsilon=5.2 \%$

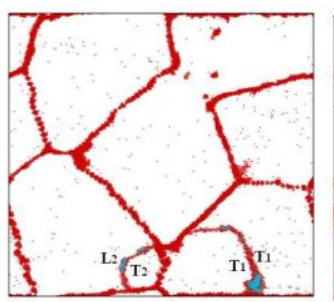

(b2) $\varepsilon=5.2 \%$

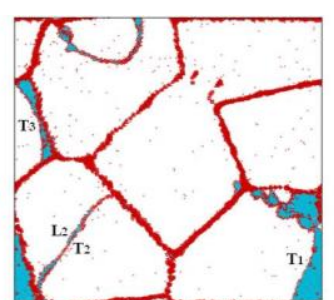

(a3) $\varepsilon=5.6 \%$

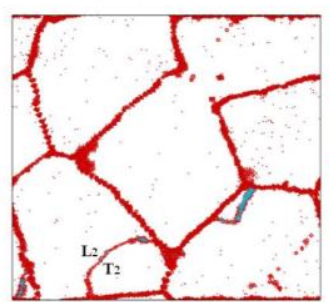

(b3) $\varepsilon=5.6 \%$

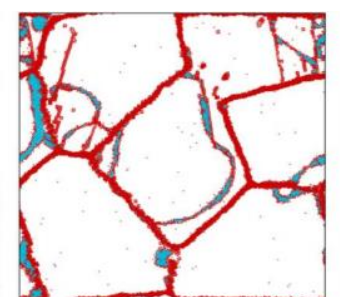

(a4) $\varepsilon=6.8 \%$

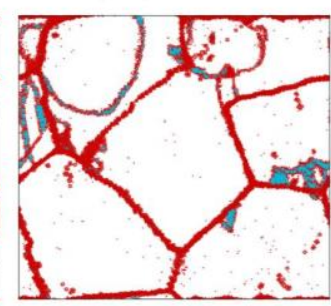

(b4) $\varepsilon=6.8 \%$

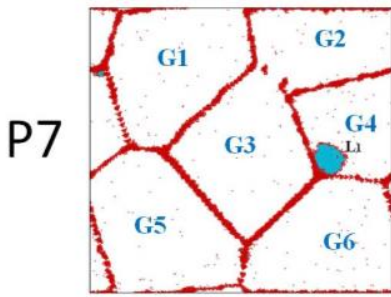

(c1) $\varepsilon=4.6 \%$

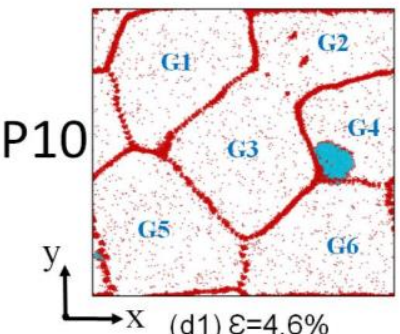

(d1) $\varepsilon=4.6 \%$

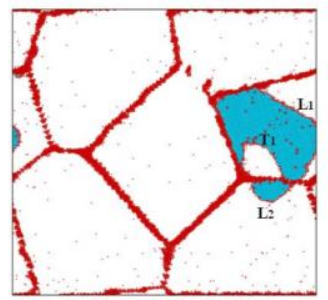

(c2) $\varepsilon=4.8 \%$

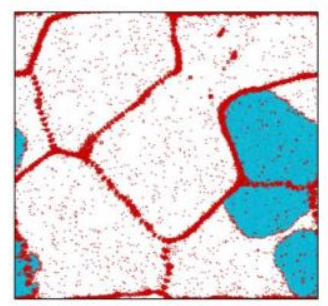

(d2) $\varepsilon=4.8 \%$

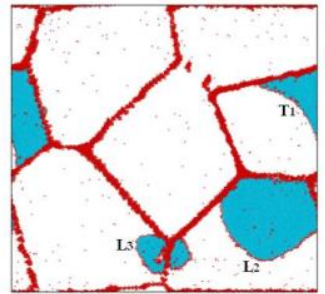

(c3) $\varepsilon=5 \%$

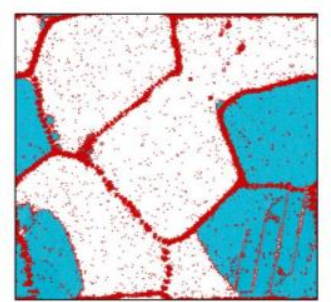

(d3) $\varepsilon=5 \%$

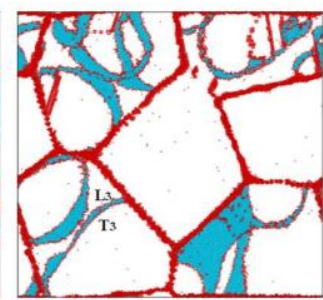

(c4) $\varepsilon=5.4 \%$

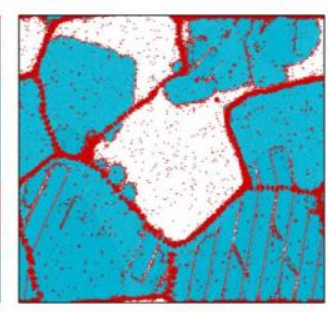

(d4) $\varepsilon=5.4 \%$

Fig.7 Snapshots of nanocrystal $\mathrm{Al}$ sample in the early stage of plastic deformation under $\mathrm{x}$-tension by different interatomic potentials. (a)-(b) corresponds to the results from the potentials of P5 and P9, (c)-(d) from the potentials of $\mathrm{P} 7$ and P10. Atoms are colored in the same manner as Fig.4, and the fcc atoms are removed.

\subsection{Deformation twinning}

Twinning was found an important deformation mechanism in metallic materials with low stacking fault energy [61], but twinning was rarely reported in the coarse-grained metals with a high stacking fault energy (e.g. Al). However, deformation twins were observed experimentally in nanocrystalline $\mathrm{Al}$ [62-64], which was regarded as a unique deformation mechanism that is different from its coarsegrained counterpart. In the present study, deformation twins were observed in G4 of the simulation sample in the case of P2 under x- and y-tension. Particularly, in the case of P10, a high density of deformation twins was observed in almost every grain. Since GBs become the primary source for the emission of partial dislocations at the nanometer scale, they are considered to be the primary mechanism for the formation of deformation twins in nanocrystalline $\mathrm{Al}$.

Fig. 8 shows the nucleation and growth of twins in G4 under tension in x-direction by P2. In Fig. 8 (a), two Shockley partial dislocations with Burger's vector $\mathbf{b}=1 / 6[2 \overline{1} 1]$ and $\mathbf{b}=1 / 6[1 \overline{1} 2]$ 
were nucleated from $\mathrm{GB}_{46}$ and $\mathrm{GB}_{24}$, respectively. The two dislocations slipped in opposite directions on the adjacent slip planes and created intrinsic stacking faults behind them. When they encountered and slipped across, the two sets of intrinsic stacking faults have one layer of atoms overlapped which generated an extrinsic stacking fault, or namely a twinning of one atomic layer, as shown in Fig.8(b) and (c). We can see that the slip of a partial dislocation leaving behind a stacking fault is the first step to the nucleation of deformation twins. Moreover, once twins were nucleated, they are very easy to grow by the nucleation and slip of new dislocations. For example, another Shockley partial dislocation with Burger's vector $\mathbf{b}=1 / 6[2 \overline{1} 1]$ was emitted from $\mathrm{GB}_{34}$ at about $14.6 \%$ tensile strain. When the new dislocation slipped on the adjacent plane of the previous twin, it led to the twin migration for one atomic layer, and thus increasing the twin distance accordingly, as shown in Fig.8(d) and (e). The surrounding GBs provided continuous nucleation source for the subsequent partial dislocations, leading to a further widening of the twinning region. In Fig.8(f), the distance between twins has increased to 4 atomic layers. The process of nucleation and growth of deformation twins was illustrated by the 'ABCABC' stacking sequences.

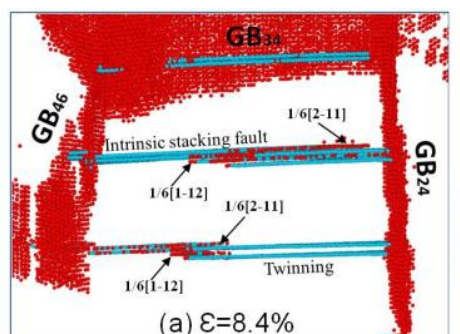

(a) $\varepsilon=8.4 \%$

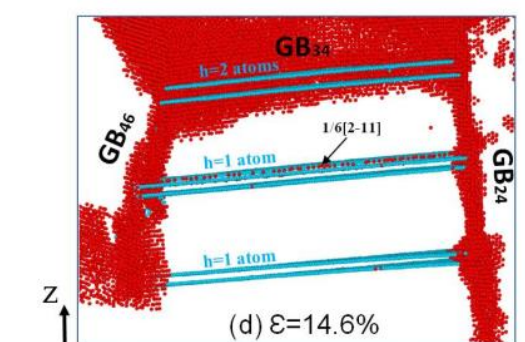

(d) $\varepsilon=14.6 \%$

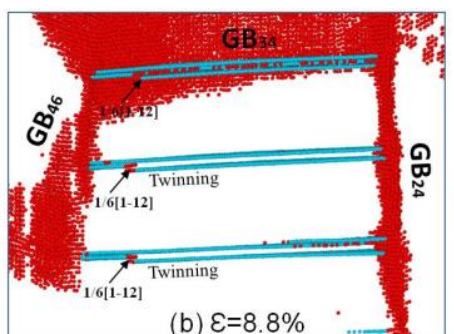

(b) $\varepsilon=8.8 \%$

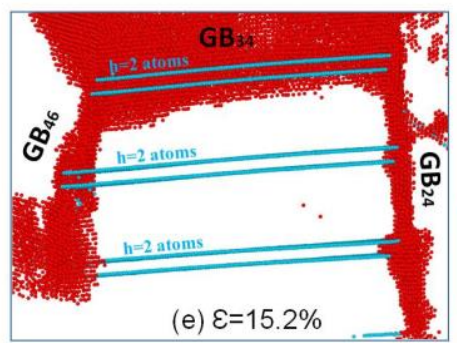

Perfect FCC structure

Intrinsic stacking fault

Twinning $(\mathrm{h}=1$ atom $)$

Twinning ( $\mathrm{h}=2$ atoms)

Twinning ( $\mathrm{h}=3$ atoms)

Twinning ( $\mathrm{h}=4$ atoms)

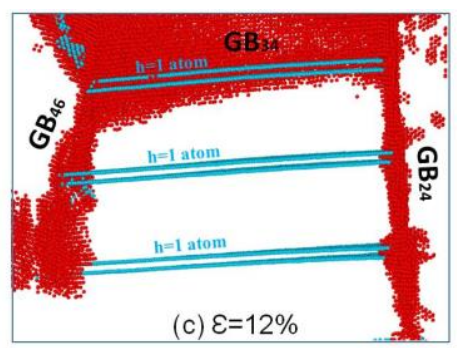

$A B C A C A C A B$ A B C A C B C A B C A B A B CA C BA B C A B C A B CACBACA B CA A B C A C B A C B C A B

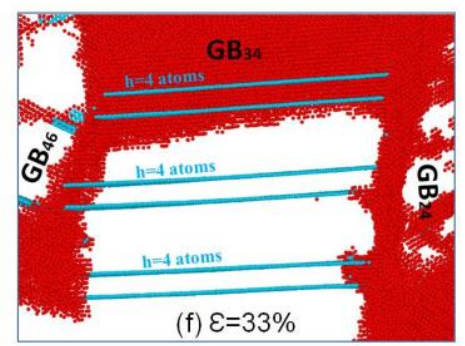

Fig.8 Dislocation and twinning process in G4 of the nanocrystalline Al sample under x-tension by P2. Atoms are colored in the same manner as Fig.4, and the fcc atoms are removed. The process of nucleation and growth of deformation twins is illustrated by the ' $\mathrm{ABCABC}$ ' stacking sequences.

Another twinning mechanism was observed via the continuous dislocation emission from the same GB. Fig. 9 shows the snapshots of G6 under tension in y-direction by P10. The first set of leading partial dislocation with Burger's vector $\mathbf{b}=1 / 6[11 \overline{2}]$ was emitted from $\mathrm{GB}_{56}$ ' at about $5.2 \%$ tensile strain. They slipped across the grain and were eventually absorbed by the $\mathrm{GB}_{36}$ and $\mathrm{GB}_{56}$ on the other side, leaving an intrinsic stacking fault across the grain. The nucleation of trailing partial dislocation was not observed with the additional tensile strain. Instead, another set of leading partials 
emitted from $\mathrm{GB}_{56}$, and they propagated on the slip plane close to the previous stacking fault plane, which led to the thickening of the stacking fault layer, as shown in Fig.9(b). Twinning occurred if the nucleated dislocations from $\mathrm{GB}_{56}$, glided on the slip plane that adjacent to the previous one, as shown in Fig.9(c). The slipping of partial dislocations on the adjacent plane turned one layer of the intrinsic stacking fault back to the perfect fcc position and created a twinning with one atomic layer. The twinning process can be also illustrated by the 'ABCABC' stacking sequences in Fig.8. However, different from the former case, all the partial dislocations were nucleated from the identical GB and they slipped in the same direction. The GB provided an effective dislocation source to emit consecutive partial dislocations and produced high density of deformation twinning in the grain, as shown in Fig.9(d).

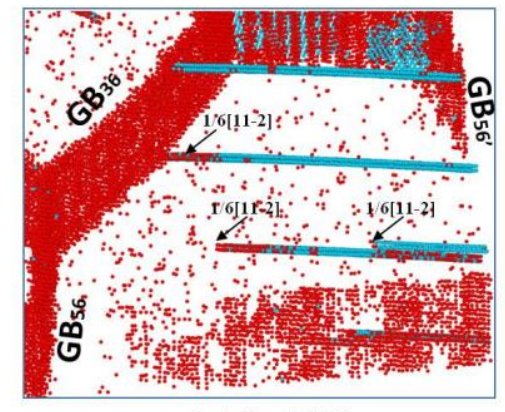

(a) $\varepsilon=5.2 \%$

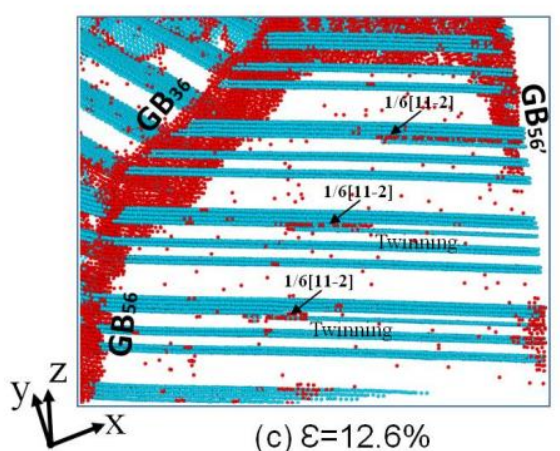

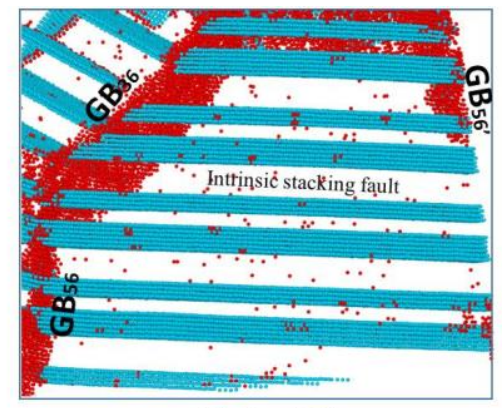

(b) $\varepsilon=10 \%$

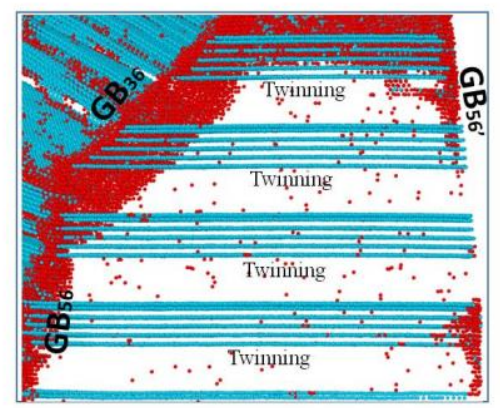

(d) $\varepsilon=19.6 \%$

Fig.9 Dislocation and twinning process in G6 of the nanocrystal Al sample under y-tension by P10. Atoms are colored in the same manner as Fig.4, and the fcc atoms are removed.

\section{Effect of interatomic potential}

We have shown in Section 4 that GB-mediated mechanisms play a dominant role in the deformation of nanocrystalline Al regardless of the selected interatomic potentials. These mechanisms include stress-driven GB migration, dislocation emission from GB, deformation twinning etc. which caused the initial plastic deformation and contributed to plastic flow, as well as crack nucleation at GBs which resulted in the intergranular fracture. Although these potentials are designed to simulate singe phase $\mathrm{Al}$ or $\mathrm{Al}$ alloys, it was found that the selection of potential strongly affected the simulation results. Table. 2 summarizes the main deformation mechanisms of nanocrystalline $\mathrm{Al}$ sample under uniaxial loading by different potentials. It is found that the dislocation activities that associated with GBs are the most common deformation mechanisms, including the nucleation and propagation of partial and extended dislocations. Since all the nucleated dislocations from GBs are Shockley type with Burger's vector $\mathbf{b}=1 / 6<112>$, to characterize the dislocation activities during deformation, the 
total length of Shockley partial dislocations as a function of tensile strain with different potentials was plotted in Fig.10. The results can be divided into three groups. In group 1, very few dislocations were detected in the cases of $\mathrm{P} 1, \mathrm{P} 2, \mathrm{P} 3$, and P4, corresponding to the mechanisms related to the self-motion of GBs, such as GB migration, GB shuffling, crack growth along GB. In group 2, a rapid growth of dislocation length was observed at the onset of plasticity, and the dislocation length reached a plateau in the stable slip stage at higher strain; this is the case of P5, P6, P7 and P9 potentials. The propagation and interaction of dislocations effectively accommodated the system stress during plastic deformation. In group 3, a higher density of dislocations was detected in the cases of P8 and P10, indicating that the nucleation of dislocations from GBs was much easier by the two potentials. The tensile tests along $\mathrm{x}$ - and $\mathrm{y}$-direction show the similar results. In the follows, we discuss how the interatomic potentials affect the microscopic deformation mechanism of the simulation samples.

Table.2 The calculated generalized stacking fault energies (GSFE) by different interatomic potentials of Al, including unstable stacking fault energy $\gamma_{u s f}$, stable stacking fault $\gamma_{s f}$, and unstable twin fault $\gamma_{u t f}$. The result from ab initio calculation based on DFT and the available experimental data (exp.) are also included for comparison. The deformation mechanisms of the simulated samples under uniaxial tension are indicated by the symbols. $(\Delta$ : primary mechanism, $\bigcirc$ : secondary mechanism, $\times$ : mechanism which is not observed or can be ignored.)

\begin{tabular}{|c|c|c|c|c|c|c|c|c|c|c|}
\hline \multirow[b]{2}{*}{$\begin{array}{c}\text { Potential } \\
\text { ID }\end{array}$} & \multirow[b]{2}{*}{$\underset{\left(\mathrm{mJ} \mathrm{m}^{-2}\right)}{\gamma_{\mathbf{u s f}}}$} & \multirow[b]{2}{*}{$\begin{array}{c}\gamma_{s f} \\
\left(\mathbf{m J} \mathbf{m}^{-2}\right)\end{array}$} & \multirow[b]{2}{*}{$\underset{\left(\mathbf{m J ~ m ^ { - 2 }}\right)}{\gamma_{\mathrm{utf}}}$} & \multirow[b]{2}{*}{$\gamma_{\text {sf }} / \gamma_{\text {usf }}$} & \multirow[b]{2}{*}{$\gamma_{\text {utf }} / \gamma_{\text {usf }}$} & \multicolumn{5}{|c|}{ Deformation mechanisms } \\
\hline & & & & & & $\begin{array}{c}\text { GB } \\
\text { migration }\end{array}$ & $\begin{array}{l}\text { Intergranular } \\
\text { fracture }\end{array}$ & $\begin{array}{c}\text { Extended } \\
\text { dislocation }\end{array}$ & $\begin{array}{c}\text { Partial } \\
\text { dislocation }\end{array}$ & $\begin{array}{c}\text { Deformation } \\
\text { twinning }\end{array}$ \\
\hline P1 & 337.1 & 187.6 & 410.8 & 0.56 & 1.22 & $\Delta$ & $\Delta$ & O & $\times$ & $\times$ \\
\hline P2 & 305.6 & 149.9 & 370.7 & 0.49 & 1.21 & $\times$ & $\times$ & $\Delta$ & $\times$ & ○ \\
\hline P3 & 242.7 & 128.5 & 299.7 & 0.53 & 1.23 & $\times$ & $\Delta$ & $\times$ & $\times$ & $\times$ \\
\hline P4 & 242.7 & 128.5 & 299.7 & 0.53 & 1.23 & $\times$ & $\Delta$ & $\times$ & $\times$ & $\times$ \\
\hline P5 & 178.0 & 143.8 & 232.7 & 0.81 & 1.31 & $\times$ & $\times$ & $\Delta$ & $\times$ & $\times$ \\
\hline P6 & 178.0 & 143.8 & 232.7 & 0.81 & 1.31 & $\times$ & $\times$ & $\Delta$ & $\times$ & $\times$ \\
\hline P7 & 127.8 & 110.5 & 160.5 & 0.86 & 1.26 & $\times$ & $\times$ & $\Delta$ & $\times$ & $\times$ \\
\hline P8 & 103.0 & 53.8 & 126.7 & 0.52 & 1.23 & O & $\times$ & $\Delta$ & $\times$ & $\times$ \\
\hline P9 & 217.8 & 172.8 & 282.1 & 0.79 & 1.29 & O & $\times$ & $\Delta$ & $x$ & $\times$ \\
\hline P10 & 75.9 & 1.2 & 76.5 & 0.02 & 1.01 & O & $\times$ & $\times$ & $\Delta$ & $\Delta$ \\
\hline $\mathrm{Ab}$ initio ${ }^{[65]}$ & 189.1 & 162.4 & 238.6 & 0.86 & 1.26 & & & & & \\
\hline Exp. & & $\begin{array}{c}135^{[66]}, 150^{[67]}, \\
166^{[68]}, 200^{[69]}\end{array}$ & & & & & & & & \\
\hline
\end{tabular}



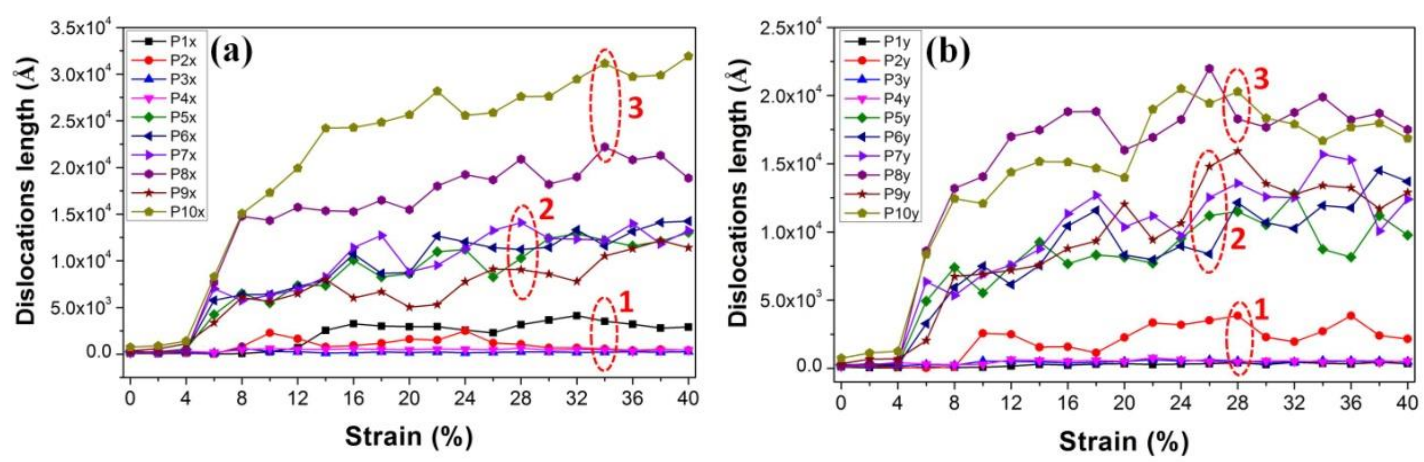

Fig.10 The length of Shockley partial dislocations as a function of tensile strain along $\mathrm{x}$-direction (a) and y-direction (b). Group 1: P1, P2, P3, and P4 potentials; Group 2: P5, P6, P7, and P9 potentials; Group 3: P8, and P10 potentials.

Stacking fault energy is the intrinsic property of materials and plays a critical role in determining the deformation behaviors of metals and their alloys [24, 25, 70, 71]. Therefore, it is instructive to calculate the generalized stacking fault energies (GSFE) of Al using different potentials, which is beneficial to understand the multiple GB-mediated mechanisms in this study. For calculating the

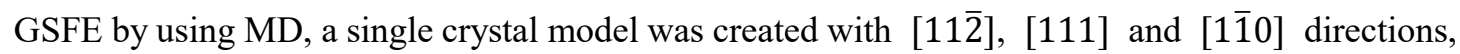
and the model was divided into the upper and lower parts. The GSFE curve was determined by rigidly displacing the upper part on (111) plane along [11̄] direction while fixing the lower block and calculating the energy change in the whole model, as illustrated in Fig.11. In step 1, the starting configuration is a perfect fcc lattice with the normal stacking of the atoms. Along the path, the atomic system first passed through an energy barrier in step 2, which is referred to unstable stacking fault energy $\left(\gamma_{u s f}\right)$. In step 3, the stable stacking fault energy $\left(\gamma_{s f}\right)$ has achieved when the displacement is about $a_{0} / \sqrt{6}\left(a_{0}\right.$ is the lattice constant). The configuration in step 3 is known as the intrinsic stacking fault. The unstable twin fault energy $\left(\gamma_{u t f}\right)$ was calculated by rigidly shifting

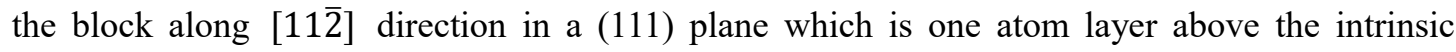
stacking fault that previously formed by rigid displacement. as shown in step 4. The GSFE curves that obtained by MD simulations with different interatomic potentials of $\mathrm{Al}$ are plotted in Fig.11, and the calculated values of $\gamma_{u s f}, \gamma_{s f}$ and $\gamma_{u t f}$ are listed in Table.2. For comparison, the result from $a b$ initio calculation based on density function theory (DFT) [65] and the available experimental data [66-69] are also included.

By comparing the GSFE values and the deformation mechanisms, it was found that $\gamma_{s f}$ plays a significant role in determining the nucleation of trailing partial dislocation and the stacking fault width. As shown in Fig.7, in the case of P9 with high value of $\gamma_{s f}\left(172.8 \mathrm{~mJ} \mathrm{~m}^{-2}\right)$, the leading and trailing partial dislocations were nucleated from GBs almost simultaneously, resulting in a very narrow stacking fault between the two partials. In the case of P5 and P7 with moderate value of $\gamma_{s f}$ (143.8 $\mathrm{mJ} \mathrm{m}^{-2}$ and $110.5 \mathrm{~mJ} \mathrm{~m}^{-2}$, respectively), the nucleation of trailing partial dislocation lagged the leading one, and there are obvious stacking faults between the two partials in the nucleation stage, although the stacking fault width decreased as the propagation of the trailing partial dislocation. In the stable slip stage, the average stacking fault width in P7 case is larger than that in P5 case because of its lower stacking fault energy. Compared to the potentials characterized by the higher value of $\gamma_{s f}$, the value calculated by potential P10 is extremely small $\left(\gamma_{s f}=1.2 \mathrm{~mJ} \mathrm{~m}^{-2}\right)$. Such a low value of $\gamma_{s f}$ implies a stable state of the stacking faults that caused by the propagation of leading partial dislocations, which significantly delayed the subsequent nucleation of the trailing 
partials. When the width of stacking fault is larger than the grain size, the trailing partial dislocations can hardly nucleated from the GB. Therefore, we observed a large number of stacking fault accumulated in the grain in the plastic deformation stage in P10 case. The result is qualitatively consistent with previous MD simulations on fcc metals with different stacking fault energies [24, 25], which also signifies the role of $\gamma_{s f}$ on the nucleation of the trailing partial dislocation. The lower the $\gamma_{s f}$ value, the more difficult to nucleate a trailing partial dislocation, and the larger the stacking fault width is. On the other hand, previous MD simulations on different fcc metals suggested that $\gamma_{s f}$ alone cannot capture the important physics of the nucleation of partial dislocations from $\mathrm{GB}$, whether the trailing partial dislocation or the extended dislocation dominates the deformation mechanism must understood in terms of the ratio $\gamma_{s f} / \gamma_{u s f}$; this value closer to unity is associated with the nucleation of extended dislocation [25]. While this is true by comparing the result of $\mathrm{P} 10$ with $\mathrm{P} 5, \mathrm{P} 7$ and $\mathrm{P} 9$ cases, we found that the stacking fault width is more sensitive to the value of $\gamma_{s f}$ than $\gamma_{s f} / \gamma_{u s f}$. For example, the value of $\gamma_{s f}$ is $\mathrm{P} 9>\mathrm{P} 5>\mathrm{P} 7>\mathrm{P} 6$ by MD simulation, so the average stacking fault width that observed in Fig. 7 is $\mathrm{P} 9<\mathrm{P} 5<\mathrm{P} 7<\mathrm{P} 6$ in the stable slip stage.
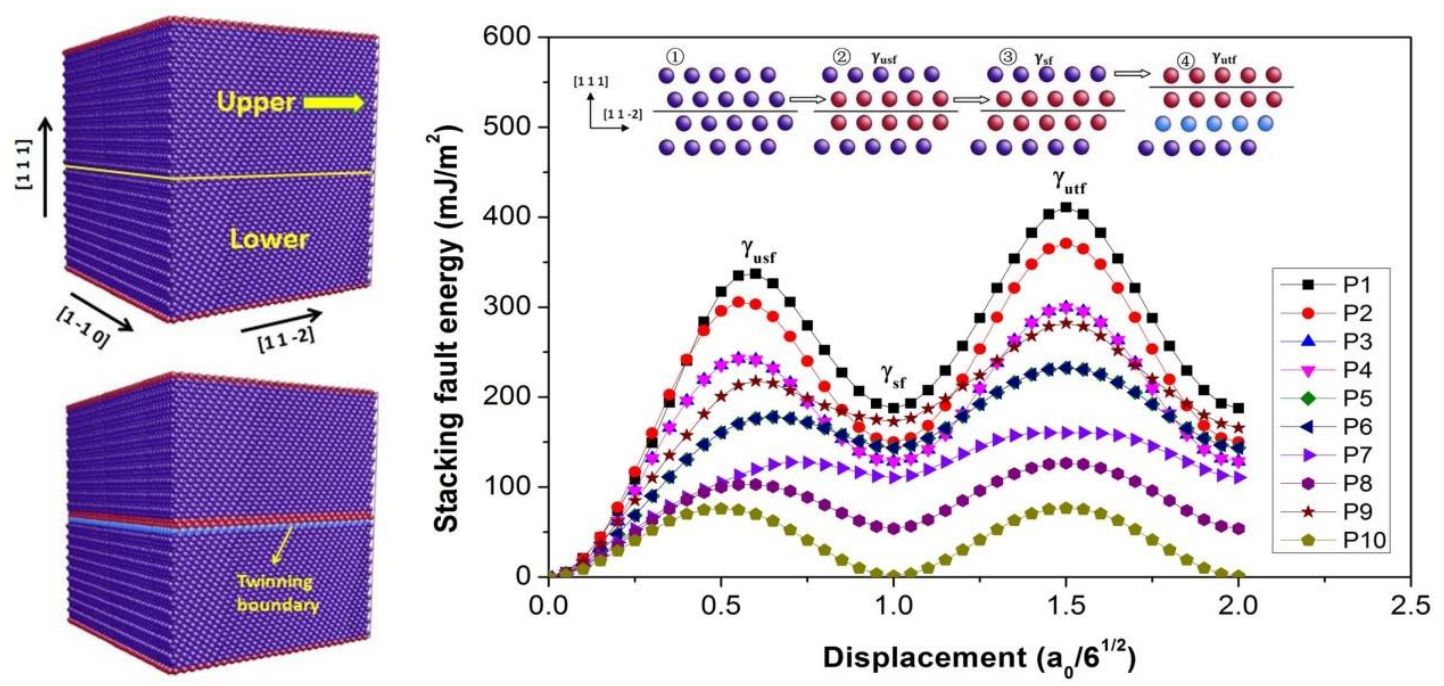

Fig.11 The stacking fault energy curve as a function of fractional displacement of Al using different interatomic potentials (P1 P10). P3 and P4, P5 and P6 show the same result. The insert atomic figures illustrate the formation of stacking fault and the twin fault, (1) perfect fcc crystal, (2) unstable stacking fault $\gamma_{u s f}$, (3) stable stacking fault $\gamma_{s f}$, and (4) unstable twin fault $\gamma_{u t f}$.

MD simulations have shown that the stacking fault, extended dislocation, and deformation twinning originated from partial dislocation from GBs. The value of $\gamma_{u s f}$ is related to the energy barrier of the nucleation of extended dislocation, and the value of $\gamma_{u t f}$ is associated with the energy barrier for twinning formation. It was reported that the ratio $\gamma_{u t f} / \gamma_{u s f}$ could control the competition of the two possible mechanisms [25]. If $\gamma_{u t f}$ is larger than $\gamma_{u s f}$, the trailing partial dislocation tends to nucleate and slip on the same plane as the leading partial to generate an extend dislocation. On the other hand, if the ratio $\gamma_{u t f} / \gamma_{u s f}$ is close to unity, the nucleation of trailing partial and its slip on the adjacent plane of the leading partial become easy, which led to the deformation twinning. It can be seen from the GSFE curves that the value of $\gamma_{u t f}$ is overall larger than $\gamma_{u s f}$ by different interatomic potentials, indicating that the nucleation of twins needs to 
overcome larger energy barrier than the nucleation of extended dislocation. In this study, deformation twins were observed occasionally in the case of $\mathrm{P} 2$, where twinning was caused by the overlapping of stacking faults that dissociated from different GBs, as shown in Fig.8. In a grain where limited dislocations were nucleated, this twinning mechanism can be regarded as small probability event. In contrast, a high density of deformation twins was observed in the case of P10. In this case, twinning was generated by the consecutive emission of partial dislocations from the same GB due to the comparable values of $\gamma_{u s f}$ and $\gamma_{u t f}\left(\gamma_{u t f} / \gamma_{u s f}=1.01\right)$. Since the stacking fault is the first step to the nucleation of deformation twins, both the values of $\gamma_{s f}$ and $\gamma_{u t f} / \gamma_{u s f}$ are necessary to give an accurate prediction of the deformation twinning.

\section{Conclusions and remarks}

MD simulations were carried out in this study to investigate the mechanical properties and deformation mechanisms of nanocrystal Al under uniaxial loading. Different interatomic potentials of $\mathrm{Al}(\mathrm{P} 1 \sim \mathrm{P} 10)$ were examined in the simulations, and they have been proved to have a significant effect on the simulation result. The main findings are concluded as follows.

(1) The stress-strain response of nanocrystal Al sample varied with the selection of interatomic potentials, including Young's modulus, yield stress, yield strain and flow stress. The Young's modulus of the simulation sample was predicted according to the elastic constants $\left(\mathrm{C}_{11}, \mathrm{C}_{12}\right.$, and $\mathrm{C}_{44}$ ) of single crystal Al obtained by the experimental measurement. Although most of the potentials give reasonable values for Young's modulus of the simulation sample, the results obtained from potential P8 and P10 are much lower than the predicted value.

(2) In the stage of plastic deformation, different GB-mediated deformation mechanisms were observed depending on the potentials, including stress-induced GB migration, crack initiation and coalescence at GB, nucleation of partial and extended dislocation from GB, and deformation twinning. These deformation mechanisms contributed to the stress flow in the plastic stage or induced the intergranular fracture. The overall mechanical behavior of the simulation sample is a result of the interplay of the multiple deformation mechanisms.

(3) The generalized stacking fault energies including the unstable stacking fault energy $\gamma_{u s f}$, the stable stacking fault $\gamma_{s f}$, and the unstable twin fault $\gamma_{u t f}$ were calculated by MD simulations using different interatomic potentials of Al. The stacking fault values were correlated to the dislocation and twinning mechanisms. The simulation result shows that $\gamma_{s f}$ is an important factor affecting the nucleation of trailing partial dislocations, and the stacking fault width is more sensitive to the value of $\gamma_{s f}$ than $\gamma_{s f} / \gamma_{u s f}$. In addition, the values of $\gamma_{s f}$ and $\gamma_{u t f} / \gamma_{u s f}$ are both necessary to give an accurate prediction of the deformation twinning. Although the simulations were performed only for $\mathrm{Al}$, the results and conclusions can be referenced to understand the properties of other materials by linking the material constants to the deformation mechanisms at the atomistic scale.

(4) The simulation result emphasized the importance of carrying out the simulation with correct interatomic potential. Improper choice of potential strongly affected the simulation results and led to some inappropriate phenomena. For example, the potential P8 and P10 cannot correctly capture the properties of nanocrystalline $\mathrm{Al}$ in terms of the mechanical responses under loading. When the potentials of P1, P3, and P4 were applied, a complete brittle fracture of the simulation sample was 
observed under tension at room temperature, which is not consistent with the actual situation. In addition, if the simulation predicts a new and surprising phenomenon that has been rarely observed previously in the experiment, the user should be careful to rule out any impact due to the choice of potential. For example, the severe coarsening of GBs and the low dislocation density at higher tensile strain by potential P2 is unusual in the plastic stage. A high density of deformation twins was observed in the simulation sample by potential P10. The abnormal mechanism is associated with the extremely low stacking fault energy that derived from the potential P10, which is contrary to the fact that $\mathrm{Al}$ is a material with high stacking fault energy.

By comparing the current simulation result and the available experimental data, we found that the potentials of P5, P6, P7, and P9 can capture the most essential mechanical properties of the simulation sample. As the nucleation of extended dislocation and GB migration are both important deformation mechanisms which are frequently reported in the previous experiments on Al, P9 can best reflect the deformation characteristics of the pure Al system. However, the choice of potential should be reconsidered if the study is turned to mechanical testing of $\mathrm{Al}$ alloy systems since the potential P5, P6 and P7 are designed specifically for Ni-Al, Ti-Al, and Al-Pb systems. Moreover, it does not mean that the potentials which gave an inappropriate result in this study are not effective or inapplicable. For examples, while the P3 and P4 potentials are not suitable for the mechanical testing in the present study, they are well designed for the simulation of solid and liquid properties of $\mathrm{Al}$ and its alloys. Also, elemental potentials taken from alloy descriptions may not work well for the pure species if the elements were fit for alloy system instead of being optimized separately; the MEAM potential (P1 and P2) is one of the examples. Therefore, the users should have some idea of what tests have been done and which properties have been evaluated for a particular potential, and deliberately choose and test the proper one to carry out the simulation according to the specific research objects and purposes.

\section{Acknowledgements}

Liang Zhang would like to acknowledge the Postdoctoral Fellowship Program (P17711) awarded by Japan Society for the Promotion of Science (JSPS) and Australian Academy of Science (AAS). This work was supported by the Grand-in-Aid for JSPS Fellows (17F17711) and the Australian Research Council Discovery Project (DP170103092).

\section{Data availability}

The data required to reproduce these findings are available from the corresponding authors upon reasonable request. 


\section{References}

[1] M.A. Meyers, A. Mishra, D.J. Benson. Mechanical properties of nanocrystalline materials, Progress in Materials Science 51 (2006) 427-556.

[2] T. Zhu, J. Li. Ultra-strength materials, Progress in Materials Science 55 (2010) 710-757.

[3] I.A. Ovid'ko, R.Z. Valiev, Y.T. Zhu. Review on superior strength and enhanced ductility of metallic nanomaterials, Progress in Materials Science 94 (2018) 462-540.

[4] E.N. Hahn, M.A. Meyers. Grain-size dependent mechanical behavior of nanocrystalline metals, Materials Science and Engineering: A 646 (2015) 101-134.

[5] Y. Mishin, M. Asta, J. Li. Atomistic modeling of interfaces and their impact on microstructure and properties, Acta Materialia 58 (2010) 1117-1151.

[6] D. Wolf, V. Yamakov, S.R. Phillpot, A. Mukherjee, H. Gleiter. Deformation of nanocrystalline materials by molecular-dynamics simulation: Relationship to experiments?, Acta Materialia 53 (2005) 140.

[7] M. Dao, L. Lu, R.J. Asaro, J.T.M. De Hosson, E. Ma. Toward a quantitative understanding of mechanical behavior of nanocrystalline metals, Acta Materialia 55 (2007) 4041-4065.

[8] X. Huang, N. Hansen, N. Tsuji. Hardening by Annealing and Softening by Deformation in Nanostructured Metals, Science 312 (2006) 249.

[9] X. Li, Y. Wei, W. Yang, H. Gao. Competing grain-boundary- and dislocation-mediated mechanisms in plastic strain recovery in nanocrystalline aluminum, Proceedings of the National Academy of Sciences 106 (2009) 16108-16113.

[10] Y. Shibuta, S. Sakane, E. Miyoshi, S. Okita, T. Takaki, M. Ohno. Heterogeneity in homogeneous nucleation from billion-atom molecular dynamics simulation of solidification of pure metal, Nature Communications 8 (2017) 10 .

[11] D. Farkas. Atomistic simulations of metallic microstructures, Current Opinion in Solid State and Materials Science 17 (2013) 284-297.

[12] L. Zhang, C. Lu, K. Tieu. A review on atomistic simulation of grain boundary behaviors in facecentered cubic metals, Computational Materials Science 118 (2016) 180-191.

[13] J. Li. AtomEye: an efficient atomistic configuration viewer, Modelling and Simulation in Materials Science and Engineering 11 (2003) 173-177.

[14] A. Stukowski. Visualization and analysis of atomistic simulation data with OVITO-the Open Visualization Tool, Modelling and Simulation in Materials Science and Engineering 18 (2010) 015012.

[15] A. Stukowski. Structure identification methods for atomistic simulations of crystalline materials, Modelling and Simulation in Materials Science and Engineering 20 (2012) 045021.

[16] A. Stukowski, K. Albe. Dislocation detection algorithm for atomistic simulations, Modelling and Simulation in Materials Science and Engineering 18 (2010) 025016. 
[17] M.S. Daw, M.I. Baskes. Semiempirical, Quantum Mechanical Calculation of Hydrogen Embrittlement in Metals, Physical Review Letters 50 (1983) 1285-1288.

[18] M.S. Daw, M.I. Baskes. Embedded-atom method: Derivation and application to impurities, surfaces, and other defects in metals, Physical Review B 29 (1984) 6443-6453.

[19] M.I. Baskes. Modified embedded-atom potentials for cubic materials and impurities, Physical Review B 46 (1992) 2727-2742.

[20] C.A. Becker, F. Tavazza, Z.T. Trautt, R.A. Buarque de Macedo. Considerations for choosing and using force fields and interatomic potentials in materials science and engineering, Current Opinion in Solid State and Materials Science 17 (2013) 277-283.

[21] http://www.ctcms.nist.gov/potentials/.

[22] http://openkim.org/.

[23] L. Zhang, C. Lu, A.K. Tieu. Nonlinear elastic response of single crystal $\mathrm{Cu}$ under uniaxial loading by molecular dynamics study, Materials Letters 227 (2018) 236-239.

[24] V. Yamakov, D. Wolf, S.R. Phillpot, A.K. Mukherjee, H. Gleiter. Deformation-mechanism map for nanocrystalline metals by molecular-dynamics simulation, Nature Materials 3 (2004) 43-47.

[25] H. Van Swygenhoven, P.M. Derlet, A.G. Frøseth. Stacking fault energies and slip in nanocrystalline metals, Nature Materials 3 (2004) 399-403.

[26] B. Valery, I.M. Mikhail, H.K. Alexander, L. Richard. Effect of stacking fault energy on mechanism of plastic deformation in nanotwinned FCC metals, Modelling and Simulation in Materials Science and Engineering 23 (2015) 055003.

[27] L. Zhang, C. Lu, Y. Shibuta. Shear response of grain boundaries with metastable structures by molecular dynamics simulations, Modelling and Simulation in Materials Science and Engineering 26 (2018) 035008 .

[28] S. Plimpton. Fast Parallel Algorithms for Short-Range Molecular Dynamics, Journal of Computational Physics 117 (1995) 1-19.

[29] M.I. Pascuet, J.R. Fernández. Atomic interaction of the MEAM type for the study of intermetallics in the Al-U alloy, Journal of Nuclear Materials 467 (2015) 229-239.

[30] B. Jelinek, S. Groh, M.F. Horstemeyer, J. Houze, S.G. Kim, G.J. Wagner, A. Moitra, M.I. Baskes. Modified embedded atom method potential for $\mathrm{Al}, \mathrm{Si}, \mathrm{Mg}, \mathrm{Cu}$, and $\mathrm{Fe}$ alloys, Physical Review B 85 (2012) 245102.

[31] M.I. Mendelev, M.J. Kramer, C.A. Becker, M. Asta. Analysis of semi-empirical interatomic potentials appropriate for simulation of crystalline and liquid $\mathrm{Al}$ and $\mathrm{Cu}$, Philosophical Magazine 88 (2008) 1723-1750.

[32] M.I. Mendelev, M. Asta, M.J. Rahman, J.J. Hoyt. Development of interatomic potentials appropriate for simulation of solid-liquid interface properties in Al-Mg alloys, Philosophical Magazine 89 (2009) 3269-3285. 
[33] Y. Mishin. Atomistic modeling of the $\gamma$ and $\gamma^{\prime}$-phases of the Ni-Al system, Acta Materialia 52 (2004) 1451-1467.

[34] R.R. Zope, Y. Mishin. Interatomic potentials for atomistic simulations of the Ti-Al system, Physical Review B 68 (2003) 024102.

[35] A. Landa, P. Wynblatt, D.J. Siegel, J.B. Adams, O.N. Mryasov, X.Y. Liu. Development of glue-type potentials for the Al-Pb system: phase diagram calculation, Acta Materialia 48 (2000) 1753-1761.

[36] X.W. Zhou, R.A. Johnson, H.N.G. Wadley. Misfit-energy-increasing dislocations in vapordeposited CoFe/NiFe multilayers, Physical Review B 69 (2004) 144113.

[37] J.M. Winey, K. Alison, Y.M. Gupta. A thermodynamic approach to determine accurate potentials for molecular dynamics simulations: thermoelastic response of aluminum, Modelling and Simulation in Materials Science and Engineering 17 (2009) 055004.

[38] J. Cai, Y.Y. Ye. Simple analytical embedded-atom-potential model including a long-range force for fcc metals and their alloys, Physical Review B 54 (1996) 8398-8410.

[39] F. Ercolessi, J.B. Adams. Interatomic Potentials from First-Principles Calculations: The ForceMatching Method, Europhysics Letters 26 (1994) 583.

[40] L. Xiang-Yang, E. Furio, B.A. James. Aluminium interatomic potential from density functional theory calculations with improved stacking fault energy, Modelling and Simulation in Materials Science and Engineering 12 (2004) 665.

[41] Y. Mishin, D. Farkas, M.J. Mehl, D.A. Papaconstantopoulos. Interatomic potentials for monoatomic metals from experimental data and ab initio calculations, Physical Review B 59 (1999) 3393-3407.

[42] J. Vallin, M. Mongy, K. Salama, O. Beckman. Elastic Constants of Aluminum, Journal of Applied Physics 35 (1964) 1825-1826.

[43] R. Hill. The Elastic Behaviour of a Crystalline Aggregate, Proceedings of the Physical Society. Section A 65 (1952) 349.

[44] S.M. Rassoulinejad-Mousavi, Y. Mao, Y. Zhang. Evaluation of copper, aluminum, and nickel interatomic potentials on predicting the elastic properties, Journal of Applied Physics 119 (2016) 244304.

[45] T.J. Rupert, D.S. Gianola, Y. Gan, K.J. Hemker. Experimental Observations of Stress-Driven Grain Boundary Migration, Science 326 (2009) 1686.

[46] T. Gorkaya, D.A. Molodov, G. Gottstein. Stress-driven migration of symmetrical $<100>$ tilt grain boundaries in Al bicrystals, Acta Materialia 57 (2009) 5396-5405.

[47] J.W. Cahn, Y. Mishin, A. Suzuki. Coupling grain boundary motion to shear deformation, Acta Materialia 54 (2006) 4953-4975.

[48] K.D. Molodov, D.A. Molodov. Grain boundary mediated plasticity: On the evaluation of grain boundary migration - shear coupling, Acta Materialia 153 (2018) 336-353.

[49] D. Farkas, S. Mohanty, J. Monk. Strain-driven grain boundary motion in nanocrystalline materials, Materials Science and Engineering: A 493 (2008) 33-40. 
[50] D.S. Gianola, S. Van Petegem, M. Legros, S. Brandstetter, H. Van Swygenhoven, K.J. Hemker. Stress-assisted discontinuous grain growth and its effect on the deformation behavior of nanocrystalline aluminum thin films, Acta Materialia 54 (2006) 2253-2263.

[51] K. Cheng, L. Zhang, C. Lu, K. Tieu. Coupled grain boundary motion in aluminium: The effect of structural multiplicity, Scientific Reports 6 (2016) 25427.

[52] L. Zhang, C. Lu, G. Michal, K. Tieu, X. Zhao, G. Deng. Influence of temperature and local structure on the shear-coupled grain boundary migration, Physica Status Solidi B 254 (2017) 1600477.

[53] L. Zhang, C. Lu, K. Tieu, Y. Shibuta. Dynamic interaction between grain boundary and stacking fault tetrahedron, Scripta Materialia 144 (2018) 78-83.

[54] L. Zhang, Y. Shibuta, C. Lu, X. Huang. Atomistic Simulation of the Interaction Between Point Defects and Twin Boundary, Physica Status Solidi B 255 (2018) 1800228.

[55] X.L. Wu, Y.T. Zhu. Partial-dislocation-mediated processes in nanocrystalline Ni with nonequilibrium grain boundaries, Applied Physics Letters 89 (2006) 031922.

[56] H. Van Swygenhoven, P.M. Derlet, A. Hasnaoui. Atomic mechanism for dislocation emission from nanosized grain boundaries, Physical Review B 66 (2002) 241011-241018.

[57] V. Yamakov, D. Wolf, S.R. Phillpot, A.K. Mukherjee, H. Gleiter. Dislocation processes in the deformation of nanocrystalline aluminium by molecular-dynamics simulation, Nature Materials 1 (2002) 45-49.

[58] L. Zhang, C. Lu, K. Tieu. Atomistic Simulation of Tensile Deformation Behavior of $\Sigma 5$ Tilt Grain Boundaries in Copper Bicrystal, Scientific Reports 4 (2014) 5919.

[59] L. Zhang, C. Lu, K. Tieu, L. Pei, X. Zhao, K. Cheng. Molecular dynamics study on the grain boundary dislocation source in nanocrystalline copper under tensile loading, Materials Research Express 2 (2015) 035009 .

[60] L. Zhang, C. Lu, K. Tieu, X. Zhao, L. Pei. The shear response of copper bicrystals with $\Sigma 11$ symmetric and asymmetric tilt grain boundaries by molecular dynamics simulation, Nanoscale 7 (2015) 7224-7233.

[61] Y.T. Zhu, X.Z. Liao, X.L. Wu. Deformation twinning in nanocrystalline materials, Progress in Materials Science 57 (2012) 1-62.

[62] X.Z. Liao, F. Zhou, E.J. Lavernia, D.W. He, Y.T. Zhu. Deformation twins in nanocrystalline Al, Applied Physics Letters 83 (2003) 5062-5064.

[63] X.Z. Liao, F. Zhou, E.J. Lavernia, S.G. Srinivasan, M.I. Baskes, D.W. He, Y.T. Zhu. Deformation mechanism in nanocrystalline Al: Partial dislocation slip, Applied Physics Letters 83 (2003) 632-634.

[64] M. Chen, E. Ma, K.J. Hemker, H. Sheng, Y. Wang, X. Cheng. Deformation Twinning in Nanocrystalline Aluminum, Science 300 (2003) 1275-1277.

[65] M. Muzyk, Z. Pakiela, K.J. Kurzydlowski. Ab initio calculations of the generalized stacking fault energy in aluminium alloys, Scripta Materialia 64 (2011) 916-918. 
[66] R.E. Smallman, P.S. Dobson. Stacking fault energy measurement from diffusion, Metallurgical Transactions 1 (1970) 2383-2389.

[67] M.J. Mills, P. Stadelmann. A study of the structure of Lomer and $60^{\circ}$ dislocations in aluminium using high-resolution transmission electron microscopy, Philosophical Magazine A 60 (1989) 355-384.

[68] L.E. Murr. Interfacial Phenomena in Metals and Alloys, Addison Wesley, Reading, MA (1975).

[69] I.L. Dillamore, R.E. Smallman. The stacking-fault energy of F.C.C. metals, Philosophical Magazine A 12 (1965) 191-193.

[70] E.B. Tadmor, S. Hai. A Peierls criterion for the onset of deformation twinning at a crack tip, Journal of the Mechanics and Physics of Solids 51 (2003) 765-793.

[71] S. Hai, E.B. Tadmor. Deformation twinning at aluminum crack tips, Acta Materialia 51 (2003) 117131. 